\title{
THE NIELSEN TYPE NUMBERS \\ FOR MAPS ON A 3-DIMENSIONAL FLAT RIEMANNIAN MANIFOLD
}

\author{
Ku Yong Ha - Jong Bum Lee
}

\begin{abstract}
Let $f: M \rightarrow M$ be a self-map on a 3-dimensional flat Riemannian $M$. We compute the Lefschetz number and the Nielsen number of $f$ by using the infra-nilmanifold structure of $M$ and the averaging formulas for the Lefschetz numbers and the Nielsen numbers of maps on infra-nilmanifolds. For each positive integer $n$, we provide an explicit algorithm for a complete computation of the Nielsen type numbers $N \mathrm{P}_{n}(f)$ and $N \Phi_{n}(f)$ of $f^{n}$.
\end{abstract}

\section{Introduction}

In dynamical systems, it is often the case that topological information can be used to study qualitative and quantitative properties of the system. For the periodic points, two Nielsen type numbers $N \mathrm{P}_{n}(f)$ and $N \Phi_{n}(f)$ are lower bounds for the number of periodic points of least period exactly $n$ and the set of periodic points of period $n$, respectively, see [9]. One can find the basic definitions, notions and some developments for the Nielsen periodic point theory in the survey articles [4], [5] and the references given there. In this paper we will determine these Nielsen type numbers of all homotopy classes of maps on a 3-dimensional flat Riemannian manifold.

2010 Mathematics Subject Classification. 57S25, 37D20, 20F18.

Key words and phrases. Flat manifold, Nielsen type numbers.

The second-named author is partially supported by the Mid-career Researcher Program through NRF grant funded by the MEST (No. 2010-0008640) and by the Sogang University Research Grant of 2010(10022). 
In order to state our main results, let us fix some notations and terminologies. Let $f: X \rightarrow X$ be a continuous self-map of a topological space $X$. We consider the following sets:

$$
\begin{aligned}
\operatorname{Fix}(f) & =\{x \in X \mid f(x)=x\}, \\
P^{n}(f) & =\operatorname{Fix}\left(f^{n}\right), \\
P_{n}(f) & =\operatorname{Fix}\left(f^{n}\right)-\bigcup_{k<n} \operatorname{Fix}\left(f^{k}\right) \\
& =\text { the set of periodic points } f \text { with least period } n .
\end{aligned}
$$

Let $\tilde{f}: \widetilde{X} \rightarrow \widetilde{X}$ be a lifting of $f$. We denote by $\varphi: \Pi \rightarrow \Pi$ the homomorphism on the deck transformation group $\Pi$ induced by the lifting $\widetilde{f}$. Namely,

$$
\varphi(\alpha) \widetilde{f}=\widetilde{f} \alpha, \quad \text { for all } \alpha \in \Pi .
$$

For each $n=1,2, \ldots, \widetilde{f}^{n}$ is a lifting of $f^{n}$, and the homomorphism determined by the lifting $\tilde{f}^{n}$ is $\varphi^{n}: \Pi \rightarrow \Pi$. The homomorphism $\varphi^{n}$ defines the Reidemeister action of $\Pi$ on $\Pi$ as follows:

$$
\Pi \times \Pi \rightarrow \Pi, \quad(\gamma, \alpha) \mapsto \gamma \alpha \varphi^{n}(\gamma)^{-1} .
$$

The Reidemeister class containing $\alpha$ will be denoted by $[\alpha]_{n}$ and the set of Reidemeister classes of $\Pi$ determined by $\varphi^{n}$ will be denoted by $\mathcal{R}\left[\varphi^{n}\right]$. The Reidemeister number $R\left(\varphi^{n}\right)$ of $\varphi^{n}$ is defined as the cardinality of $\mathcal{R}\left[\varphi^{n}\right]$.

The Reidemeister number $R(f)$ of the continuous map $f$ is defined as the Reidemeister number $R(\varphi)$ of an induced homomorphism $\varphi$. Note that the Reidemeister number $R(f)$ does not depend on the particular choice of the lifting $\tilde{f}$ and hence on the particular choice of the induced homomorphism $\varphi$. Also the fixed point classes do not depend on the choice of liftings, although the corresponding Reidemeister classes may.

Let $O_{n}(\varphi)$ be the number of irreducible, essential periodic point orbits of $\mathcal{R}\left[\varphi^{n}\right]$. If $[\alpha]^{n}$ is irreducible and essential, then so is the corresponding periodic point class $\mathbb{F}$ and its $f$-orbit contains at least $n$ periodic points of least period $n$.

The prime Nielsen-Jiang periodic number of period $n$ is defined by the formula

$$
N \mathrm{P}_{n}(f)=n \times O_{n}(\varphi) .
$$

Take the set of all the essential orbits, of any period $m \mid n$, which do not contain any essential orbits of lower period. To each such an orbit, find the lowest period which it can be reduced to. The full Nielsen-Jiang periodic number of period $n$, denoted by $N \Phi_{n}(f)$, is the sum of these numbers.

Then the Nielsen type numbers $N \mathrm{P}_{n}(f)$ and $N \Phi_{n}(f)$ are homotopy invariant, non-negative integers [9, Theorem III.4.10]. Therefore,

$$
N \mathrm{P}_{n}(f) \leq \min \left\{\left|P_{n}(g)\right| \mid g \simeq f\right\}, \quad N \Phi_{n}(f) \leq \min \left\{\left|P^{n}(g)\right| \mid g \simeq f\right\} .
$$


In this paper when the topological space $X$ is a 3-dimensional flat Riemannian manifold, we will determine these two homotopy invariants for all maps on $X$. This will be the first complete computation on a 3-dimensional infra-nilmanifold.

In Section 2, we will consider 3-dimensional flat Riemannian manifolds which can be considered as 3-dimensional analogues of the 2-dimensional Klein bottle, and show that they have solvmanifold structures. In Section 3, we will consider one of such manifolds and devote ourselves to compute these homotopy invariants. This is a continuation of the work done on the Klein bottle [12]. However, the computations involved in this paper are much complicated compared to the previous ones. We will refer to [12] and the references given there for necessary preliminaries and facts.

\section{3-dimensional flat Riemannian manifolds}

We have a complete classification of 3-dimensional crystallographic groups. Such a group $\Pi$ has an explicit representation $\Pi \rightarrow \mathbb{R}^{3} \rtimes \mathrm{GL}(3, \mathbb{Z})$ (not into $\left.\mathbb{R}^{3} \rtimes \mathrm{O}(3)\right)$ in the book [1].

There are 3-dimensional analogues of the classical 2-dimensional Klein bottle $K^{2}$. In fact, among ten 3-dimensional Bieberbach groups, there are three 3dimensional Bieberbach groups $\Pi$ with holonomy group $\mathbb{Z}_{2}$. These are orientable $2 / 1 / 1 / 02$, and non-orientable $2 / 2 / 1 / 02$ and $2 / 2 / 2 / 02$. The bold-faced numbers associated to the 3 -dimensional Bieberbach groups refer to the numbering in the book [1]. Indeed, it is easy to see that these are $\mathfrak{G}_{2}, \mathfrak{B}_{1}$ and $\mathfrak{B}_{2}$ in $[16$, Theorems 3.5.5 and 3.5.9], respectively. Write

$$
\begin{array}{rlrl}
e_{1} & =\left[\begin{array}{l}
1 \\
0 \\
0
\end{array}\right], & e_{2}=\left[\begin{array}{l}
0 \\
1 \\
0
\end{array}\right], & e_{3}=\left[\begin{array}{l}
0 \\
0 \\
1
\end{array}\right] ; \\
a_{1}=\left[\begin{array}{c}
0 \\
1 / 2 \\
0
\end{array}\right], & A_{1}=\left[\begin{array}{rrr}
-1 & 0 & 0 \\
0 & 1 & 0 \\
0 & 0 & -1
\end{array}\right] ; \\
a_{2}=\left[\begin{array}{c}
0 \\
0 \\
1 / 2
\end{array}\right], & A_{2}=\left[\begin{array}{ccc}
1 & 0 & 0 \\
0 & -1 & 0 \\
0 & 0 & 1
\end{array}\right] \\
a_{3}=\left[\begin{array}{ccc}
0 \\
0 \\
1 / 2
\end{array}\right], & A_{3}=\left[\begin{array}{lll}
0 & 1 & 0 \\
1 & 0 & 0 \\
0 & 0 & 1
\end{array}\right]
\end{array}
$$

Then $\alpha_{i}=\left(a_{i}, A_{i}\right)$ and $t_{i}=\left(e_{i}, I_{3}\right)$ be elements of $\mathbb{R}^{3} \rtimes \operatorname{GL}(3, \mathbb{R})$ and $A_{i}$ has period 2 . 
Furthermore,

$$
\begin{array}{lll}
\alpha_{1}^{2}=t_{2}, & t_{1} \alpha_{1} t_{1}^{-1}=\alpha_{1} t_{1}^{-2}, & t_{3} \alpha_{1} t_{3}^{-1}=\alpha_{1} t_{3}^{-2}, \\
\alpha_{2}^{2}=t_{3}, & t_{1} \alpha_{2} t_{1}^{-1}=\alpha_{2}, & t_{2} \alpha_{2} t_{2}^{-1}=\alpha_{2} t_{2}^{-2}, \\
\alpha_{3}^{2}=t_{3}, & t_{1} \alpha_{3} t_{1}^{-1}=\alpha_{3} t_{1}^{-1} t_{2}, & t_{2} \alpha_{3} t_{2}^{-1}=\alpha_{3} t_{1} t_{2}^{-1} .
\end{array}
$$

Let $\Gamma$ be the integral matrices of $\mathbb{R}^{3}$. Then it forms a lattice of $\mathbb{R}^{3}$ and $\Gamma \backslash \mathbb{R}^{3}$ is the 3 -torus. It is easy to check that the subgroup

$$
\Pi_{i}=\left\langle\Gamma,\left(a_{i}, A_{i}\right)\right\rangle \subset \mathbb{R}^{3} \rtimes \mathrm{GL}(3, \mathbb{R})
$$

generated by the lattice $\Gamma$ and the element $\alpha_{i}=\left(a_{i}, A_{i}\right)$ is discrete and torsion free, and has $\Gamma$ as a normal subgroup of index 2. Thus $\Pi_{i}$ is a 3 -dimensional Bieberbach group and the quotient space $\Pi_{i} \backslash \mathbb{R}^{3}$ is a 3-dimensional flat manifold which is orientable when $i=1$ and non-orientable when $i=2$ or 3 . The projection $\Gamma \backslash \mathbb{R}^{3} \rightarrow \Pi_{i} \backslash \mathbb{R}^{3}$ is a double covering projection. We shall denote the flat manifold $\Pi_{i} \backslash \mathbb{R}^{3}$ by $K_{i}$, and the torus $\Gamma \backslash \mathbb{R}^{3}$ by $T$. Note also that

$$
\begin{aligned}
& \text { 2/1/1/02: } \Pi_{1}=\left\langle t_{1}, \alpha_{1}, t_{3} \mid t_{1} \alpha_{1}=\alpha_{1} t_{1}^{-1}, t_{3} \alpha_{1}=\alpha_{1} t_{3}^{-1},\left[t_{1}, t_{3}\right]=1\right\rangle, \\
& \text { 2/2/1/02: } \Pi_{2}=\left\langle t_{1}, t_{2}, \alpha_{2} \mid t_{1} \alpha_{2}=\alpha_{2} t_{1}, t_{2} \alpha_{2}=\alpha_{2} t_{2}^{-1},\left[t_{1}, t_{2}\right]=1\right\rangle, \\
& \text { 2/2/2/02: } \Pi_{3}=\left\langle t_{1}, t_{2}, \alpha_{3} \mid t_{1} \alpha_{3}=\alpha_{3} t_{2}, t_{2} \alpha_{3}=\alpha_{3} t_{1},\left[t_{1}, t_{2}\right]=1\right\rangle .
\end{aligned}
$$

Furthermore, since we can embed $\operatorname{Aff}(3)=\mathbb{R}^{3} \rtimes \operatorname{GL}(3, \mathbb{R})$ in $\operatorname{GL}(4, \mathbb{R})$ as

$$
\operatorname{Aff}(3)=\left\{\left[\begin{array}{ll}
A & \mathbf{x} \\
0 & 1
\end{array}\right] \mid A \in \mathrm{GL}(3, \mathbb{R}), \mathbf{x} \in \mathbb{R}^{3}\right\} \subset \mathrm{GL}(4, \mathbb{R}),
$$

we can embed each $\Pi_{i}$ in $\operatorname{GL}(4, \mathbb{R})$ so that

$$
t_{i}=\left[\begin{array}{cc}
I_{3} & e_{i} \\
0 & 1
\end{array}\right], \quad \alpha_{i}=\left[\begin{array}{cc}
A_{i} & a_{i} \\
0 & 1
\end{array}\right] \in \mathrm{GL}(4, \mathbb{R}) .
$$

Observe that the $\Pi_{i}$ are non-nilpotent, 2-step solvable groups

$$
\Pi_{1}=\mathbb{Z}^{2} \rtimes_{\phi_{1}} \mathbb{Z}, \quad \Pi_{2}=\mathbb{Z}^{2} \rtimes_{\phi_{2}} \mathbb{Z}, \quad \Pi_{3}=\mathbb{Z}^{2} \rtimes_{\phi_{3}} \mathbb{Z}
$$

where

$$
\phi_{1}=\left[\begin{array}{rr}
-1 & 0 \\
0 & -1
\end{array}\right], \quad \phi_{2}=\left[\begin{array}{rr}
1 & 0 \\
0 & -1
\end{array}\right], \quad \phi_{3}=\left[\begin{array}{ll}
0 & 1 \\
1 & 0
\end{array}\right] .
$$

Since each $\phi_{i}$ has an eigenvalue -1 , the solvmanifold with fundamental group $\Pi_{i}$ is not an $\mathcal{N} \mathcal{R}$-solvmanifold (see [10], [15] for the definition of $\mathcal{N} \mathcal{R}$-solvmanifolds).

Proposition 2.1. The flat manifolds $K_{i}$ are compact solvmanifolds.

Proof. Consider the simply connected solvable Lie group $G_{1}=\mathbb{C}^{2} \rtimes_{\sigma_{1}} \mathbb{R}$ where $\sigma_{1}(t)$ is the rotation by $2 \pi t$ on each factor of $\mathbb{C}$, namely, $\sigma_{1}(t):\left(z_{1}, z_{2}\right) \mapsto$ $\left(e^{2 \pi i t} z_{1}, e^{2 \pi i t} z_{2}\right)$. Let $H_{1}$ be the closed subgroup of $G_{1}$ given by

$$
H_{1}=\left\{(m+i x, n+i y, k / 2) \in G_{1} \mid m, n, k \in \mathbb{Z}, x, y \in \mathbb{R}\right\} .
$$


Then it is easily seen that the compact solvmanifold $H_{1} \backslash G_{1}$ is homeomorphic to the flat manifold $K_{1}$.

Note that $\Pi_{2}=\left\langle t_{1}\right\rangle \times\left\langle t_{2}, \alpha_{2}\right\rangle$ is isomorphic to the product of the infinite cyclic group $\mathbb{Z}$ and the 2 -dimensional Klein bottle group $\pi_{1}(K)$. This yields that the flat manifold $K_{2}$ is homeomorphic to the product space $S^{1} \times K^{2}$. Hence $K_{2}$ is a compact solvmanifold. As above, we can consider the simply connected solvable Lie group $G_{2}=\mathbb{R} \times\left(\mathbb{C} \rtimes_{\sigma_{2}} \mathbb{R}\right)=\mathbb{R} \times \widetilde{E}_{0}(2)$ where $\sigma_{2}(t)$ is the rotation by $2 \pi t$ on $\mathbb{C}$, namely, $\sigma_{2}(t): z \mapsto e^{2 \pi i t} z$. Let $H_{2}$ be the closed subgroup of $G_{2}$ given by

$$
H_{2}=\left\{(m, n+i x, k / 2) \in G_{2} \mid m, n, k \in \mathbb{Z}, x \in \mathbb{R}\right\} .
$$

Then we can see that the compact solvmanifold $H_{2} \backslash G_{2}$ is homeomorphic to the product $S^{1} \times K^{2}$ and hence to the flat manifold $K_{2}$.

Consider the simply connected solvable Lie group $G_{3}=\mathbb{C}^{3} \rtimes_{\sigma_{3}} \mathbb{R}$ where $\sigma_{3}(t)$ is given by the composition (or product) of three matrices

$$
\begin{aligned}
& {\left[\begin{array}{lllllc}
1 & 0 & 0 & 0 & 0 & 0 \\
0 & 1 & 0 & 0 & 0 & 0 \\
0 & 0 & 1 & 0 & 0 & 0 \\
0 & 0 & 0 & 1 & 0 & 0 \\
0 & 0 & 0 & 0 & \cos 2 \pi t & -\sin 2 \pi t \\
0 & 0 & 0 & 0 & \sin 2 \pi t & \cos 2 \pi t
\end{array}\right],} \\
& {\left[\begin{array}{cccccc}
\cos 4 \pi t & 0 & -\sin 4 \pi t & 0 & 0 & 0 \\
0 & \cos 4 \pi t & 0 & 0 & -\sin 4 \pi t & 0 \\
\sin 4 \pi t & 0 & \cos 4 \pi t & 0 & 0 & 0 \\
0 & 0 & 0 & 1 & 0 & 0 \\
0 & \sin 4 \pi t & 0 & 0 & \cos 4 \pi t & 0 \\
0 & 0 & 0 & 0 & 0 & 1
\end{array}\right],} \\
& {\left[\begin{array}{ccccccc}
\cos 2 \pi t & 0 & -\sin 2 \pi t & 0 & 0 & 0 \\
0 & \cos 2 \pi t & 0 & -\sin 2 \pi t & 0 & 0 \\
\sin 2 \pi t & 0 & \cos 2 \pi t & 0 & 0 & 0 \\
0 & \sin 2 \pi t & 0 & \cos 2 \pi t & 0 & 0 \\
0 & 0 & 0 & 0 & \cos 2 \pi t & -\sin 2 \pi t \\
0 & 0 & 0 & 0 & \sin 2 \pi t & \cos 2 \pi t
\end{array}\right]}
\end{aligned}
$$

Let $H_{3}$ be the closed subgroup of $G_{3}$ given by

$$
H_{3}=\left\{(x+i m, y+i n, u+i v, k / 4) \in G_{3} \mid k, m, n \in \mathbb{Z}, x, y, u, v \in \mathbb{R}\right\} .
$$

Then it is easily seen that the compact solvmanifold $H_{3} \backslash G_{3}$ is homeomorphic to the flat manifold $K_{3}$.

For a compact solvmanifold $K=H \backslash G$ where $S$ is a connected, simply connected solvable Lie group and $H$ is a closed uniform subgroup of $G$, let $N$ be the nilradical of $G$; then $N$ fits a short exact sequence

$$
0 \longrightarrow N \longrightarrow G \longrightarrow G / N \cong \mathbb{R}^{s} \longrightarrow 0
$$


The closed subgroup $H$ of $G$ yields a uniform subgroup $N \cap H$ of $N$. Moreover, the closed subgroup $H$ of $G$ induces a short exact sequence $0 \rightarrow N \cap H \rightarrow H \rightarrow$ $H / N \cap H \cong H \cdot N / N \rightarrow 0$ so that the following diagram is commutative

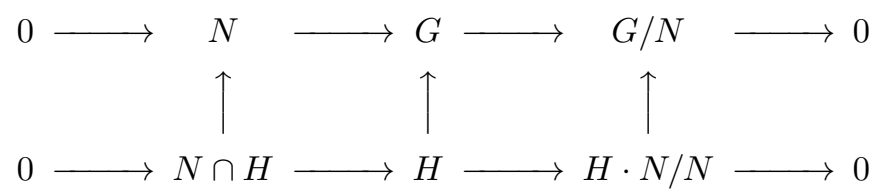

This gives rise to the fibration, called the Mostow fibration,

$$
N \cap H \backslash N \longrightarrow K=H \backslash G \longrightarrow H \cdot N \backslash G
$$

over a torus base $H \cdot N \backslash G$ with compact nilmanifold fiber $N \cap H \backslash N$, see [15], [2]. It is known that the Mostow fibration is orientable if and only if the solvmanifold $K$ is a nilmanifold, see [15, Lemma 3.1].

For $G_{1}=\mathbb{C}^{2} \rtimes_{\sigma_{1}} \mathbb{R}$, we have that

- the nilradical of $G_{1}$ is $N_{1}=\mathbb{C}^{2}$,

- $N_{1} \cap H_{1}=\left\{(m+i x, n+i y, 0) \in G_{1} \mid m, n \in \mathbb{Z}, x, y \in \mathbb{R}\right\}$,

- $N_{1} \cdot H_{1}=\left\{\left(\mathbb{C}^{2}, k / 2\right) \mid k \in \mathbb{Z}\right\}$.

Thus the Mostow fibration has the base the circle with $\alpha_{1}$ as a generator of the fundamental group, and the fiber the torus with $t_{1}$ and $t_{3}$ as generators of the fundamental group. Notice here that $K_{1}$ is orientable as a manifold, however the Mostow fibration structure on $K_{1}$ is not orientable. In this sense, $K_{1}$ is a 3-dimensional analogue of the classical 2-dimensional Klein bottle.

It is clear that $K_{2}=S^{1} \times K^{2}$. The Mostow fibration is the product of the trivial bundle over $S^{1}$ with the standard fibration of the Klein bottle $K^{2}$.

For $G_{3}=\mathbb{C}^{3} \rtimes_{\sigma_{3}} \mathbb{R}$, we have that

- the nilradical of $G_{3}$ is $N_{3}=\mathbb{C}^{3}$,

- $N_{3} \cap H_{3}=\left\{(x+i m, y+i n, u+i v, 0) \in G_{3} \mid m, n \in \mathbb{Z}, x, y, u, v \in \mathbb{R}\right\}$,

- $N_{3} \cdot H_{3}=\left\{\left(\mathbb{C}^{3}, k / 4\right) \mid k \in \mathbb{Z}\right\}$.

Thus the Mostow fibration has the base the circle with $\alpha_{3}$ as a generator of the fundamental group, and the fiber the torus with $t_{1}$ and $t_{2}$ as generators of the fundamental group.

\section{The Nielsen type numbers of maps on $K$}

Now let us recall some of the main results in [6], [7], [8].

Definition 3.1. The map $f: M \rightarrow M$ is called weakly Jiang if either $N(f)=0$ or $N(f)=R(f)$.

Theorem 3.2 ([6, Theorem 1], [7, Theorems 1.2]). Let $f: M \rightarrow M$ be a selfmap of a nilmanifold or $\mathcal{N} \mathcal{R}$ solvmanifold, or if $M$ is an arbitrary solvmanifold 
suppose that $f^{n}$ is weakly Jiang. If $N\left(f^{n}\right) \neq 0$, then for all $m \mid n$

$$
N\left(f^{m}\right)=\sum_{k \mid m} N \mathrm{P}_{k}(f), \quad N \mathrm{P}_{m}(f)=\sum_{k \mid m} \mu(k) N\left(f^{m / k}\right),
$$

where $\mu$ is the Möbius function.

Theorem 3.3 ([7, Corollary 4.6]). Let $f: M \rightarrow M$ be a self-map. If $M$ is a solvmanifold, then

$$
N \Phi_{n}(f)=\sum_{k \mid n} N \mathrm{P}_{k}(f), \quad N \mathrm{P}_{n}(f)=\sum_{k \mid n} \mu(k) N \Phi_{n / k}(f) .
$$

Since our flat manifolds $K_{i}$ are solvmanifolds, according to Theorem 3.3 it is enough to find the formula for the prime Nielsen-Jiang periodic number or the full Nielsen-Jiang periodic number. Note that $K_{i}$ are not $\mathcal{N} \mathcal{R}$ solvmanifolds. When $f^{n}$ is weakly Jiang and $N\left(f^{n}\right) \neq 0$ we can find the formula easily using Theorem 3.2. However, the remaining cases are not rare and are required a lot of efforts to work, see for example [12].

In this paper we shall consider the flat manifold $K_{1}$ only and evaluate the Nielsen type numbers for all self-maps on $K_{1}$. We believe the results of this case are worth recording once and for all. For simplicity we will use the notation $K$ and $\Pi$ for the manifold $K_{1}$ and its fundamental group $\Pi_{1}$.

\section{Self-maps on $K$}

Note that $\Pi$ fits a short exact sequence

$$
0 \longrightarrow \mathbb{Z}^{2} \longrightarrow \Pi \longrightarrow \mathbb{Z} \longrightarrow 0
$$

where $s_{1}$ and $s_{2}$ are generators of the normal subgroup $\mathbb{Z}^{2}$ and $\alpha$ is a generator of the quotient group $\mathbb{Z}$ so that $\alpha$ acts on $s_{i}$ by $\alpha: s_{i} \mapsto s_{i}^{-1}$. Of course, we can embed $\Pi$ into $\mathbb{R}^{3} \rtimes \operatorname{GL}(3, \mathbb{R})$ by the assignment $s_{1} \mapsto t_{1}, s_{2} \mapsto t_{2}$ and $\alpha \mapsto \alpha_{1}$ where

$$
t_{i}=\left(e_{i}, I_{3}\right), \quad \alpha_{1}=\left(\left[\begin{array}{c}
0 \\
0 \\
1 / 2
\end{array}\right],\left[\begin{array}{rrr}
-1 & 0 & 0 \\
0 & -1 & 0 \\
0 & 0 & 1
\end{array}\right]\right)
$$

Note that the group $\left\langle t_{1}, t_{2}, \alpha_{1}\right\rangle$ is conjugate to the 3 -dimensional Bieberbach group 2/1/1/02 in the book [1] by

$$
\left(\left[\begin{array}{l}
0 \\
0 \\
0
\end{array}\right],\left[\begin{array}{lll}
1 & 0 & 0 \\
0 & 0 & 1 \\
0 & 1 & 0
\end{array}\right]\right) \in \mathbb{R} \rtimes \mathrm{GL}(3, \mathbb{R}) .
$$


Lemma 4.1. Any homomorphism $\varphi: \Pi \rightarrow \Pi$ is given as follows:

$$
\begin{aligned}
& \varphi\left(s_{1}\right)=s_{1}^{\left(a_{11}-(-1)^{\omega} a_{11}\right) / 2} s_{2}^{\left(a_{21}-(-1)^{\omega} a_{21}\right) / 2}, \\
& \varphi\left(s_{2}\right)=s_{1}^{\left(a_{12}-(-1)^{\omega} a_{12}\right) / 2} s_{2}^{\left(a_{22}-(-1)^{\omega} a_{22}\right) / 2}, \\
& \varphi(\alpha)=s_{1}^{b_{1}} s_{2}^{b_{2}} \alpha^{\omega} .
\end{aligned}
$$

Proof. Every element of $\Pi$ can be written uniquely as $s_{1}^{\ell} s_{2}^{m} \alpha^{n}$. Thus

$$
\varphi\left(s_{1}\right)=s_{1}^{a_{11}} s_{2}^{a_{21}} \alpha^{\omega_{1}}, \quad \varphi\left(s_{2}\right)=s_{1}^{a_{12}} s_{2}^{a_{22}} \alpha^{\omega_{2}}, \quad \varphi(\alpha)=s_{1}^{b_{1}} s_{2}^{b_{2}} \alpha^{\omega},
$$

for some integers $a_{i j}, b_{i}, \omega_{i}$ and $\omega$. Since $\alpha s_{i} \alpha^{-1}=s_{i}^{-1}$ implies $\alpha=s_{i} \alpha s_{i}$, the equations

$$
\varphi(\alpha)=\varphi\left(s_{i}\right) \varphi(\alpha) \varphi\left(s_{i}\right)
$$

yields that

$$
\begin{aligned}
s_{1}^{b_{1}} s_{2}^{b_{2}} \alpha^{\omega} & =\left(s_{1}^{a_{1 i}} s_{2}^{a_{2 i}} \alpha^{\omega_{i}}\right)\left(s_{1}^{b_{1}} s_{2}^{b_{2}} \alpha^{\omega}\right)\left(s_{1}^{a_{1 i}} s_{2}^{a_{2 i}} \alpha^{\omega_{i}}\right) \\
& =s_{1}^{a_{1 i}+(-1)^{\omega_{i}} b_{1}+(-1)^{\omega_{i}+\omega} a_{1 i}} s_{2}^{a_{2 i}+(-1)^{\omega_{i}} b_{2}+(-1)^{\omega_{i}+\omega} a_{2 i}} \alpha^{2 \omega_{i}+\omega}
\end{aligned}
$$

or

$$
\omega_{i}=0 \quad \text { and } \quad\left(1+(-1)^{\omega}\right) a_{i j}=0 \quad \text { for } 1 \leq i, j \leq 2 .
$$

The equation $\varphi\left(s_{1}\right) \varphi\left(s_{2}\right)=\varphi\left(s_{2}\right) \varphi\left(s_{1}\right)$ is redundant!

Explicitly we have:

Corollary 4.2. Any homomorphism $\varphi: \Pi \rightarrow \Pi$ is given as follows:

(a) When $\omega$ is odd,

$$
\varphi\left(s_{1}\right)=s_{1}^{a_{11}} s_{2}^{a_{21}}, \quad \varphi\left(s_{2}\right)=s_{1}^{a_{12}} s_{2}^{a_{22}}, \quad \varphi(\alpha)=s_{1}^{b_{1}} s_{2}^{b_{2}} \alpha^{\omega} .
$$

(b) When $\omega$ is even,

$$
\varphi\left(s_{1}\right)=1, \quad \varphi\left(s_{2}\right)=1, \quad \varphi(\alpha)=s_{1}^{b_{1}} s_{2}^{b_{2}} \alpha^{\omega} .
$$

Immediately we have:

Corollary 4.3. The group of pure translations in $\Pi, \Gamma=\left\langle s_{1}, s_{2}, s_{3}\right\rangle$, is a fully invariant subgroup of $\Pi$.

Let $f: K \rightarrow K$ be a continuous map on the flat manifold $K=\Pi \backslash \mathbb{R}^{3}$ and choose a lifting $\tilde{f}: \mathbb{R}^{3} \rightarrow \mathbb{R}^{3}$ of $f$. The lifting $\tilde{f}$ induces a homomorphism $\varphi: \Pi \rightarrow \Pi$ which is defined by the following rule:

$$
\varphi(\gamma) \circ \tilde{f}=\tilde{f} \circ \gamma \quad \text { for all } \gamma \in \Pi \text {. }
$$

Given $f$, we consider another lifting of $f$. It is of the form $\beta \circ \widetilde{f}$ for some $\beta \in \Pi$, and the homomorphism on $\Pi$ induced by $\beta \circ \tilde{f}$ is $\tau_{\beta} \circ \varphi$. Indeed, for all $\gamma \in \Pi$,

$$
(\beta \circ \tilde{f}) \circ \gamma=\beta \circ \varphi(\gamma) \circ \tilde{f}=\left(\beta \circ \varphi(\gamma) \circ \beta^{-1}\right) \circ(\beta \circ \tilde{f})=\left(\tau_{\beta} \circ \varphi\right)(\gamma) \circ(\beta \circ \tilde{f}) .
$$

Now we describe $\tau_{\beta} \circ \varphi$. 
A homomorphism $\varphi$ given as in Corollary 4.2 is called of type $(F, \mathbf{b}, \omega)$ where $F$ and $\mathbf{b}$ are integer matrices

$$
F=\left[\begin{array}{ll}
a_{11} & a_{12} \\
a_{21} & a_{22}
\end{array}\right], \quad \mathbf{b}=\left[\begin{array}{l}
b_{1} \\
b_{2}
\end{array}\right] .
$$

When $\omega$ is even, $F$ is the zero matrix. A self-map on $K$ is called of type $(F, \mathbf{b}, \omega)$ if it induces a homomorphism of type $(F, \mathbf{b}, \omega)$. Since the element $\beta$ of $\Pi$ is of the form $\beta=s_{1}^{\ell} s_{2}^{m} \alpha^{n}$ for some integers $\ell, m$ and $n$, we have:

(1) When $\omega$ is odd, then

$$
\begin{aligned}
& \tau_{\beta} \circ \varphi\left(s_{i}\right)=s_{1}^{(-1)^{n} a_{1 i}} s_{2}^{(-1)^{n} a_{2 i}}, \quad(i=1,2), \\
& \tau_{\beta} \circ \varphi(\alpha)=s_{1}^{2 \ell+(-1)^{n} b_{1}} s_{2}^{2 m+(-1)^{n} b_{2}} \alpha^{\omega} .
\end{aligned}
$$

Thus we can choose a lifting $\tilde{f}$ of $f$ so that (i) $F$ is unique up to $\pm I$ and (ii) $b_{1}, b_{2} \in\{0,1\}$.

(2) When $\omega$ is even, then

$$
\begin{aligned}
& \tau_{\beta} \circ \varphi\left(s_{i}\right)=1, \quad(i=1,2), \\
& \tau_{\beta} \circ \varphi(\alpha)=s_{1}^{(-1)^{n} b_{1}} s_{2}^{(-1)^{n} b_{2}} \alpha^{\omega} .
\end{aligned}
$$

Thus we can choose a lifting $\tilde{f}$ of $f$ so that $b_{1} \geq 0$; when $b_{1}=0$ then $b_{2} \geq 0$.

Consequently, we can choose and then fix a lifting $\tilde{f}$ of $f$ so that the induced homomorphism $\varphi$ as in Corollary 4.2 satisfies the following: when $\omega$ is odd, (i) $F$ is unique up to $\pm I$ and (ii) $b_{1}, b_{2} \in\{0,1\}$, and when $\omega$ is even, $b_{1} \geq 0$; when $b_{1}=0$ then $b_{2} \geq 0$. Such a homomorphism $\varphi: \Pi \rightarrow \Pi$ is called of normalized type $(F, \mathbf{b}, \omega)$. A self-map $f$ on $\Pi \backslash \mathbb{R}^{3}$ is said to be of normalized type $(F, \mathbf{b}, \omega)$ if it induces a homomorphism of normalized type $(F, \mathbf{b}, \omega)$.

Suppose $f$ and $f^{\prime}$ are homotopic maps on the flat manifold $K$. It is well known that the induced homomorphisms $\varphi$ and $\varphi^{\prime}$ are conjugate by an element of $\Pi$. It follows that $\varphi=\varphi^{\prime}$ as normalized type. Note that when $\omega$ is even $F=0$.

Conversely suppose $f$ and $f^{\prime}$ are maps on $K$ so that the homomorphisms $\varphi$ and $\varphi^{\prime}$ are the same (as normalized type). Since $K$ is an aspherical manifold, it is well-known that $f$ and $f^{\prime}$ are homotopic, i.e. such a map is unique up to homotopy.

In all, we have obtained the following homotopy classification of maps on the flat manifold $K$.

THEOREM 4.4. Every continuous maps on the flat manifold $K$ is homotopic to a map of normalized type $(F, \mathbf{b}, \omega)$. Furthermore, two such maps of (normalized) type are homotopic if and only if they have the same normalized types. 
Notation 4.5. A self-map $f: K \rightarrow K$ on the flat manifold $K$ of type $(F, \mathbf{b}, \omega)$ will be denoted by $f_{(F, \mathbf{b}, \omega)}$.

Lemma 4.6. Every self-map $f: K \rightarrow K$ of type $(F, \mathbf{b}, \omega)$ has an affine endomorphism $(\delta, D) \in \mathbb{R}^{3} \rtimes \operatorname{Endo}\left(\mathbb{R}^{3}\right)$ as a homotopy lifting given by

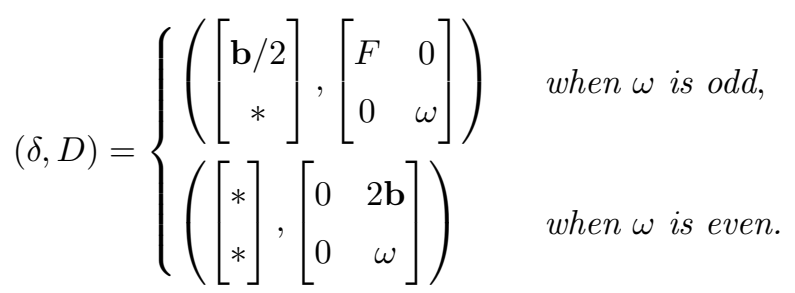

Conversely, such an affine map $(\delta, D)$ induces a map on $K$ of type $(F, \mathbf{b}, \omega)$.

Proof. Let $f: K \rightarrow K$ be a self-map inducing a homomorphism $\varphi$ on $\Pi$ of type $(F, \mathbf{b}, \omega)$. Due to [14, Theorem 1.1], there exists an affine endomorphism $(\delta, D) \in \mathbb{R}^{3} \rtimes \operatorname{Endo}\left(\mathbb{R}^{3}\right)$ such that

$$
\begin{aligned}
& \varphi\left(s_{i}\right)(\delta, D)=(\delta, D) s_{i} \quad(i=1,2), \\
& \varphi(\alpha)(\delta, D)=(\delta, D) \alpha .
\end{aligned}
$$

These equalities yield the formula for $(\delta, D)$ by a simple computation. Furthermore, the above equalities imply that $(\delta, D)$ induces a map $\bar{f}$ on $K$, i.e. $\bar{f}$ has a lifting $(\delta, D)$. Hence $f$ and $\bar{f}$ induce the same homomorphism $\varphi$. Since $K$ is a $K(\pi, 1)$-manifold, $\bar{f}$ is homotopic to $f$.

Definition 4.7. Let $f$ be a self-map on $K$ of type $(F, \mathbf{b}, \omega)$. The linear part $D$ of a homotopy lifting of $f$ in Lemma 4.6 is called the linearization of $f$. We call $F$ the fiber of $f$.

REMARK 4.8. Let $f$ be a self-map on $K$ of type $(F, \mathbf{b}, \omega)$ with a lifting $\tilde{f}$ which induces the homomorphism $\varphi$. Then $\tilde{f}^{n}$ is a lifting of $f^{n}$ and the corresponding homomorphism is $\varphi^{n}$. It can seen easily that

$$
\begin{aligned}
\varphi^{n}\left(s_{i}\right)(\delta, D)^{n} & =(\delta, D)^{n} s_{i} \quad(i=1,2), \\
\varphi^{n}(\alpha)(\delta, D)^{n} & =(\delta, D)^{n} \alpha .
\end{aligned}
$$

Thus $D^{n}$ is the linearization of $f^{n}$, and $F^{n}$ is the fiber of $f^{n}$. Note also that the integer $\omega$ for $f$ becomes $\omega^{n}$ for $f^{n}$.

Theorem 4.9. Let $f: K \rightarrow K$ be a self-map of type $(F, \mathbf{b}, \omega)$. Then for any positive integer $n$,

$$
N\left(f^{n}\right)=\frac{1}{2}\left|1-\omega^{n}\right|\left(\left|\operatorname{det}\left(I-F^{n}\right)\right|+\left|\operatorname{det}\left(I+F^{n}\right)\right|\right) .
$$

In particular when $\omega$ is even $N\left(f^{n}\right)=\left|1-\omega^{n}\right|$. 
Proof. We recall the averaging formula for the Nielsen number on infranilmanifolds from [11, Theorem 3.5] and [13, Theorem 1.4]

$$
N(f)=\frac{1}{|\Psi|} \sum_{A \in \Psi}\left|\operatorname{det}\left(A_{*}-f_{*}\right)\right| .
$$

Thus for the case of the flat manifold $K$, we have $\Psi=\left\langle A_{1}\right\rangle \cong \mathbb{Z}_{2}$ and

$$
\begin{aligned}
N\left(f^{n}\right) & =\frac{1}{2}\left(\left|\operatorname{det}\left(I-D^{n}\right)\right|+\left|\operatorname{det}\left(A_{1}-D^{n}\right)\right|\right) \\
& =\frac{1}{2}\left|1-\omega^{n}\right|\left(\left|\operatorname{det}\left(I-F^{n}\right)\right|+\left|\operatorname{det}\left(I+F^{n}\right)\right|\right) .
\end{aligned}
$$

Remark that if $\lambda_{i}$ is an eigenvalue of $F$ then $1 \pm \lambda_{i}$ is an eigenvalue of $I \pm F$. Hence

$$
\begin{aligned}
\operatorname{det}\left(I \pm F^{n}\right) & =\left(1 \pm \lambda_{1}^{n}\right)\left(1 \pm \lambda_{2}^{n}\right)=1+\lambda_{1}^{n} \lambda_{2}^{n} \pm\left(\lambda_{1}^{n}+\lambda_{2}^{n}\right) \\
& =1+\operatorname{det}\left(F^{n}\right) \pm \operatorname{tr}\left(F^{n}\right) .
\end{aligned}
$$

\section{Weakly Jiang maps on $K$}

Let $f$ be a self-map on $K$ of normalized type $(F, \mathbf{b}, \omega)$ with induced homomorphism $\varphi$ on $\Pi$. By Lemma $4.6, f^{n}$ is of type $\left(F^{n}, \mathbf{b}_{n}, \omega^{n}\right)$, not necessarily normalized, where

$$
\mathbf{b}_{n}= \begin{cases}\left(I+F+\ldots+F^{n-1}\right) \mathbf{b} & \text { when } \omega \text { is odd; } \\ \omega^{n-1} \mathbf{b} & \text { when } \omega \text { is even. }\end{cases}
$$

We will discuss the case where $f^{n}$ is weakly Jiang.

Theorem 5.1. Let $f$ be a self-map on $K$ of type $(F, \mathbf{b}, \omega)$. Then $f^{n}$ is weakly Jiang if and only if one of the following holds:

(a) Case $N\left(f^{n}\right)=0$ :

- $\operatorname{det}\left(I \pm F^{n}\right)=0$,

- $\omega=1$,

- $\omega=-1$ and $n$ even.

(b) Case $N\left(f^{n}\right)=R\left(f^{n}\right)$ :

- $\omega$ even,

- $\omega=-1, n$ odd and $\operatorname{det}\left(I \pm F^{n}\right) \neq 0$,

- $\omega \neq \pm 1$ odd and $\operatorname{det}\left(I \pm F^{n}\right) \neq 0$.

Proof. The first case follows directly from Theorem 4.9. For the second case, we shall recall the averaging formula for the Reidemeister coincidence number on orientable infra-nilmanifolds from [3, Theorem 6.11]. We can use this result because our infra-nilmanifold $K$ is orientable. In fact, the fixed point 
version of this result is true for all infra-nilmanifolds. Namely,

$$
R(f)=\frac{1}{|\Psi|} \sum_{A \in \Psi} \sigma\left(\operatorname{det}\left(A_{*}-f_{*}\right)\right),
$$

where $\sigma: \mathbb{R} \rightarrow \mathbb{R} \cup\{\infty\}$ is defined by $\sigma(0)=\infty$ and $\sigma(x)=|x|$ for all $x \neq 0$. Hence

$$
R\left(f^{n}\right)=\frac{1}{2}\left(\sigma\left(\operatorname{det}\left(I-D^{n}\right)\right)+\sigma\left(\operatorname{det}\left(A-D^{n}\right)\right)\right) .
$$

Therefore, $N\left(f^{n}\right)=R\left(f^{n}\right)$ if and only if $\operatorname{det}\left(I-D^{n}\right) \neq 0$ and $\operatorname{det}\left(A-D^{n}\right) \neq 0$ if and only if $\omega^{n} \neq 1, \operatorname{det}\left(I-F^{n}\right) \neq 0 \neq \operatorname{det}\left(I+F^{n}\right)$.

Immediately we have:

Corollary 5.2. If $N\left(f^{n}\right)=R\left(f^{n}\right)$ holds, then for any $m$ with $m \mid n$, we have that $N\left(f^{m}\right)=R\left(f^{m}\right)$.

Proof. Observe that if $m \mid n$ then $I-F^{n}=\left(I-F^{m}\right)\left(I+F^{m}+\ldots+F^{n-m}\right)$ and so $\operatorname{det}\left(I-F^{m}\right) \mid \operatorname{det}\left(I-F^{n}\right)$; moreover, in addition, if $n / m$ is even then $I-F^{n}=\left(I+F^{m}\right)\left(I-F^{m}+\ldots-F^{n-m}\right)$ implies that $\operatorname{det}\left(I+F^{m}\right) \mid \operatorname{det}\left(I-F^{n}\right)$, and if $n / m$ is odd then $I+F^{n}=\left(I+F^{m}\right)\left(I-F^{m}+\ldots+F^{n-m}\right)$ implies that $\operatorname{det}\left(I+F^{m}\right) \mid \operatorname{det}\left(I+F^{n}\right)$. This implies our result.

Corollary 5.3. If $\operatorname{det}\left(I \pm F^{n}\right)=0$ holds, then $n$ must be odd and the eigenvalues of $F$ are \pm 1 . Moreover, for any odd $m \operatorname{det}\left(I \pm F^{m}\right)=0$ holds and, for any even $m, \operatorname{det}\left(I-F^{m}\right)=0$ and $\operatorname{det}\left(I+F^{m}\right)=4$ and so $N\left(f^{m}\right)=2\left|1-\omega^{m}\right|$.

Proof. Assume $\operatorname{det}\left(I \pm F^{n}\right)=0$. Let $\lambda_{1}$ and $\lambda_{2}$ be the eigenvalues of $F$. Then $1+\lambda_{1}^{n} \lambda_{2}^{n}-\left(\lambda_{1}^{n}+\lambda_{2}^{n}\right)=0$ and $1+\lambda_{1}^{n} \lambda_{2}^{n}+\left(\lambda_{1}^{n}+\lambda_{2}^{n}\right)=0$. These identities induce that $\lambda_{2}^{n}=-\lambda_{1}^{n}$ and $\lambda_{1}^{2 n}=1$. If the $\lambda_{i}$ are real then $\lambda_{1}=-\lambda_{2}= \pm 1$ and $n$ must be odd; thus in this case for any odd $m$, we must have $\operatorname{det}\left(I \pm F^{m}\right)=0$. If the $\lambda_{i}$ are complex numbers (so that $\lambda_{2}=\bar{\lambda}_{1}$ ) then $\lambda_{2}^{n}=-\lambda_{1}^{n}$ yields that $\lambda_{2}^{n}=$ $\bar{\lambda}_{1}^{n}=-\lambda_{1}^{n}$ and thus $\lambda_{1}^{n}= \pm i$ and $\lambda_{1}^{2 n}=( \pm i)^{2}=-1$, which is a contradiction. Now it is simply a routine to check the last assertion.

Let $f$ be a self-map on $K$ with induced homomorphism $\varphi$ on $\Pi$ of type $(F, \mathbf{b}, \omega)$. Assuming $N\left(f^{n}\right)=R\left(f^{n}\right)$ so that $f^{n}$ is weakly Jiang, we will find a complete set of representatives for the Reidemeister set $\mathcal{R}\left[\varphi^{n}\right]$.

Notation 5.4. Note that for any $k, \varphi^{k}$ acts on the integral lattice spanned by $s_{1}$ and $s_{2}$ by matrix multiplication by $F^{k}$. That is, the exponents of $\varphi^{k}\left(s_{1}^{i_{1}} s_{2}^{i_{2}}\right)$ are the entries of $F^{k}$ multiplied by the column vector $\mathbf{i}$ of $\left(i_{1}, i_{2}\right)$. For simplicity, we will use the following notations:

$$
s^{\mathbf{i}}:=s_{1}^{i_{1}} s_{2}^{i_{2}}, \quad s^{F^{k} \mathbf{i}}:=\varphi^{k}\left(s^{\mathbf{i}}\right) .
$$

Similarly, we will use $\overline{\mathbf{i}}$ and $F^{k} \overline{\mathbf{i}}$ for the elements in a quotient group of $\mathbb{Z}^{2}$ represented by $\mathbf{i}$ and $F^{k} \mathbf{i}$, respectively. 
Notation 5.5. As we are dealing with only integral matrices $M$ of order 2, when we say the image of $M, \operatorname{Im}(M)$, we shall mean that

$$
\operatorname{Im}(M)=\text { image }\left\{M: \mathbb{Z}^{2} \rightarrow \mathbb{Z}^{2}\right\} .
$$

REMARK 5.6 ( $\omega$ even). Let $f$ be a self-map on $K$ with induced homomorphism $\varphi$ on $\Pi$ of type $(F, \mathbf{b}, \omega)$ where $\omega$ is even and so $F=0$. In this case $R\left(\varphi^{n}\right)=N\left(f^{n}\right)=\left|1-\omega^{n}\right| \neq 0$. Since

$$
\varphi^{n}\left(s^{\mathbf{x}} \alpha^{y}\right)=s^{y \omega^{n-1} \mathbf{b}} \alpha^{y \omega^{n}}, \quad \varphi^{n}\left(s^{\mathbf{x}} \alpha^{y}\right)^{-1}=s^{-y \omega^{n-1} \mathbf{b}} \alpha^{-y \omega^{n}},
$$

we have for each $\mathbf{i} \in \mathbb{Z}^{2}$ and $j \in \mathbb{Z}$

$$
\left(s^{\mathbf{x}} \alpha^{y}\right)\left(s^{\mathbf{i}} \alpha^{j}\right) \varphi^{n}\left(s^{\mathbf{x}} \alpha^{y}\right)^{-1}=s^{\mathbf{x}+(-1)^{y} \mathbf{i}-(-1)^{y+j} y \omega^{n-1} \mathbf{b}} \alpha^{y\left(1-\omega^{n}\right)+j} .
$$

It follows that $\mathcal{R}\left[\varphi^{n}\right]=\left\{[\alpha]_{n},\left[\alpha^{2}\right]_{n}, \ldots,\left[\alpha^{\left|1-\omega^{n}\right|}\right]_{n}\right\}$.

In fact, $\varphi: \Pi \rightarrow \Pi$ induces the following commutative diagram

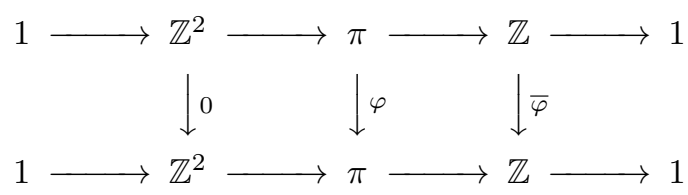

This diagram induces the exact sequence of the Reidemeister sets

$$
\mathcal{R}(0) \longrightarrow \mathcal{R}(\varphi) \longrightarrow \mathcal{R}(\bar{\varphi}) \longrightarrow 1 \text {. }
$$

Since $\mathcal{R}(0)=1$ and the same holds for all iterations $\varphi^{k}$, we get the equality of all prime and full Nielsen-Jiang numbers. Thus the results are the same as in the corresponding self-maps of the circle, the base space of the fibration. This was suggested by the referee.

REMARK 5.7 ( $\omega=-1$ and $n$ odd). Let $f$ be a self-map on $K$ with induced homomorphism $\varphi$ on $\Pi$ of type $(F, \mathbf{b}, \omega)$ where $\omega=-1, n$ odd. In this case $N\left(f^{n}\right)=\left|\operatorname{det}\left(I-F^{n}\right)\right|+\left|\operatorname{det}\left(I+F^{n}\right)\right|$. Note also that $\varphi^{n}$ is of type $\left(F^{n}, \mathbf{b}_{n},-1\right)$. Observe that

$$
\varphi^{n}\left(s^{\mathbf{x}} \alpha^{y}\right)= \begin{cases}s^{F^{n} \mathbf{x}+\mathbf{b}_{n}} \alpha^{-y} & \text { when } y \text { is odd } \\ s^{F^{n} \mathbf{x}} \alpha^{-y} & \text { when } y \text { is even }\end{cases}
$$

and so

$$
\varphi^{n}\left(s^{\mathbf{x}} \alpha^{y}\right)^{-1}= \begin{cases}s^{F^{n} \mathbf{x}+\mathbf{b}_{n}} \alpha^{y} & \text { when } y \text { is odd; } \\ s^{-F^{n} \mathbf{x}} \alpha^{y} & \text { when } y \text { is even. }\end{cases}
$$

For each $\mathbf{i} \in \mathbb{Z}^{2}$ and $j \in \mathbb{Z}$, we then have

$$
\left(s^{\mathbf{x}} \alpha^{y}\right)\left(s^{\mathbf{i}} \alpha^{j}\right) \varphi^{n}\left(s^{\mathbf{x}} \alpha^{y}\right)^{-1}= \begin{cases}s^{\mathbf{x}-\mathbf{i}-(-1)^{j}\left(F^{n} \mathbf{x}+\mathbf{b}_{n}\right)} \alpha^{2 y+j} & \text { when } y \text { is odd } \\ s^{\mathbf{x}+\mathbf{i}-(-1)^{j}\left(F^{n} \mathbf{x}\right)} \alpha^{2 y+j} & \text { when } y \text { is even. }\end{cases}
$$

Now we analyze this identity more as follows: 
CASE $1 . j \equiv 0$ or $2 \bmod 4$.

We choose $y$ to be even or odd respectively and then we can assume $j=0$. The exponents of $s_{1}$ and $s_{2}$ are then, respectively,

$$
\left(I-F^{n}\right) \mathbf{x}+\mathbf{i} \quad \text { or } \quad\left(I-F^{n}\right) \mathbf{x}-\mathbf{i}-\mathbf{b}_{n} .
$$

CASE 2. $j \equiv 1$ or $3 \bmod 4$.

We choose $y$ to be even or odd, respectively, and then we can assume $j=1$. The exponents of $s_{1}$ and $s_{2}$ are then, respectively

$$
\left(I+F^{n}\right) \mathbf{x}+\mathbf{i} \text { or }\left(I+F^{n}\right) \mathbf{x}-\mathbf{i}+\mathbf{b}_{n} .
$$

Since $\operatorname{det}\left(I \pm F^{n}\right) \neq 0$, we have that $\operatorname{Im}\left(I-F^{n}\right)$ and $\operatorname{Im}\left(I+F^{n}\right)$ have finite indices in $\mathbb{Z}^{2}$, and $\left|\mathbb{Z}^{2} / \operatorname{Im}\left(I \pm F^{n}\right)\right|=\left|\operatorname{det}\left(I \pm F^{n}\right)\right|$.

Consequently, we can choose a complete set of representatives of the Reidemeister classes $\left[s^{\mathbf{i}} \alpha^{j}\right]_{n}$ in the quotient groups $\mathbb{Z}^{2} / \operatorname{Im}\left(I \pm F^{n}\right)$. Namely,

$$
\mathcal{R}\left[\varphi^{n}\right]=\left\{\left[s^{\overline{\mathbf{i}}}\right]_{n} \mid \overline{\mathbf{i}} \in \mathbb{Z}^{2} / \operatorname{Im}\left(I-F^{n}\right)\right\} \bigcup\left\{\left[s^{\overline{\mathbf{i}}} \alpha\right]_{n} \mid \overline{\mathbf{i}} \in \mathbb{Z}^{2} / \operatorname{Im}\left(I+F^{n}\right)\right\} .
$$

When $\operatorname{det}\left(I \pm F^{n}\right) \neq 0$, we have that $\operatorname{Im}\left(I-F^{n}\right)$ and $\operatorname{Im}\left(I+F^{n}\right)$ have finite indices in $\mathbb{Z}^{2}$, and $\left|\mathbb{Z}^{2} / \operatorname{Im}\left(I \pm F^{n}\right)\right|=\left|\operatorname{det}\left(I \pm F^{n}\right)\right|$. Thus in this case $f^{n}$ is weakly Jiang.

REMARK $5.8(\omega \neq \pm 1$ odd). Let $f$ be a self-map on $K$ with induced homomorphism $\varphi$ on $\Pi$ of type $(F, \mathbf{b}, \omega)$ where $\omega$ is odd, $\omega \neq \pm 1$. In this case

$$
N\left(f^{n}\right)=\frac{1}{2}\left|1-\omega^{n}\right|\left(\left|\operatorname{det}\left(I-F^{n}\right)\right|+\left|\operatorname{det}\left(I+F^{n}\right)\right|\right) .
$$

Note also that $\varphi^{n}$ is of type $\left(F^{n}, \mathbf{b}_{n}, \omega^{n}\right)$. Observe that

$$
\varphi^{n}\left(s^{\mathbf{x}} \alpha^{y}\right)= \begin{cases}s^{F^{n} \mathbf{x}+\mathbf{b}_{n}} \alpha^{y \omega^{n}} & \text { when } y \text { is odd; } \\ s^{F^{n} \mathbf{x}} \alpha^{y \omega^{n}} & \text { when } y \text { is even, }\end{cases}
$$

and so

$$
\varphi^{n}\left(s^{\mathbf{x}} \alpha^{y}\right)^{-1}= \begin{cases}s^{F^{n} \mathbf{x}+\mathbf{b}_{n}} \alpha^{-y \omega^{n}} & \text { when } y \text { is odd } \\ s^{-F^{n} \mathbf{x}} \alpha^{-y \omega^{n}} & \text { when } y \text { is even }\end{cases}
$$

For each $\mathbf{i} \in \mathbb{Z}^{2}$ and $j \in \mathbb{Z}$, we then have

$$
\left(s^{\mathbf{x}} \alpha^{y}\right)\left(s^{\mathbf{i}} \alpha^{j}\right) \varphi^{n}\left(s^{\mathbf{x}} \alpha^{y}\right)^{-1}= \begin{cases}s^{\mathbf{x}-\mathbf{i}-(-1)^{j}\left(F^{n} \mathbf{x}+\mathbf{b}_{n}\right)} \alpha^{y\left(1-\omega^{n}\right)+j} & \text { when } y \text { is odd; } \\ s^{\mathbf{x}+\mathbf{i}-(-1)^{j} F^{n} \mathbf{x}} \alpha^{y\left(1-\omega^{n}\right)+j} & \text { when } y \text { is even }\end{cases}
$$

Noting that $1-\omega^{n}$ is even, we will consider this identity according to $j$ modulo $2\left(1-\omega^{n}\right)$. Let $j \equiv j^{\prime}$ or $j \equiv j^{\prime}+\left|1-\omega^{n}\right|$ so that $0 \leq j^{\prime}<\left|1-\omega^{n}\right|$. We choose $y$ to be even or odd respectively and then we can assume $j=j^{\prime}$. The exponents of $s_{1}$ and $s_{2}$ are then, respectively,

$$
\left(I-(-1)^{j^{\prime}} F^{n}\right) \mathbf{x}+\mathbf{i} \quad \text { or } \quad\left(I-(-1)^{j^{\prime}} F^{n}\right) \mathbf{x}-\mathbf{i}-(-1)^{j^{\prime}} \mathbf{b}_{n} .
$$


This implies that

$$
\begin{aligned}
\mathcal{R}\left[\varphi^{n}\right]= & \left\{\left[s^{\overline{\mathbf{i}}} \alpha^{j}\right]_{n}\left|\overline{\mathbf{i}} \in \mathbb{Z}^{2} / \operatorname{Im}\left(I-F^{n}\right) ; j=0,2, \ldots,\right| 1-\omega^{n} \mid-2\right\} \\
& \cup\left\{\left[s^{\overline{\mathbf{i}}} \alpha^{j}\right]_{n}\left|\overline{\mathbf{i}} \in \mathbb{Z}^{2} / \operatorname{Im}\left(I+F^{n}\right) ; j=1,3, \ldots,\right| 1-\omega^{n} \mid-1\right\} .
\end{aligned}
$$

\section{The Nielsen type numbers: non-weakly Jiang case I}

Now we will evaluate the Nielsen type number of periodic points of $f$ in the case when $f^{n}$ is not weakly Jiang, i.e. $0 \neq N\left(f^{n}\right) \neq R\left(f^{n}\right)$. Explicitly, we should consider the following two cases in this subsection and the next subsection:

- $n$ is odd, $\omega=-1$, and exactly one of $I-F^{n}$ and $I+F^{n}$ has zero determinant.

- $\omega \neq \pm 1$ is odd and exactly one of $I-F^{n}$ and $I+F^{n}$ has zero determinant.

Notation 6.1. Let $n=p_{0}^{e_{0}} \ldots p_{t}^{e_{t}}$ be the prime decomposition of the positive integer $n$ so that $p_{0}=2$ with $e_{0} \geq 0$ and the other $p_{j}$ 's are distinct odd primes. Then a proper maximal divisor $m$ of $n$ is of the form $n / p_{j}$. Write for each $j=0, \ldots, t$ and for each nonempty subset $\left\{k_{0}, \ldots, k_{s}\right\} \subset\{0, \ldots, t\}$,

$$
n_{j}=\frac{n}{p_{j}} \quad \text { and } \quad\left(n_{k_{0}}, \ldots, n_{k_{s}}\right)=\operatorname{gcd}\left(n_{k_{0}}, \ldots, n_{k_{s}}\right)=\frac{n}{p_{k_{0}} \ldots p_{k_{s}}}
$$

Before computing the Nielsen type numbers, we observe the following necessary and elementary facts.

LEMMA 6.2. If $F$ is any integer square matrix and $p, q$ are relatively prime numbers, then

$$
\begin{aligned}
\operatorname{Im}\left(I+F^{p}+\ldots+F^{p q-p}\right) \cap \operatorname{Im}\left(I+F^{q}+\ldots+F^{p q-q}\right) & \\
& =\operatorname{Im}\left(I+F+\ldots+F^{p q-1}\right) .
\end{aligned}
$$

ProOF. The following observation

$$
\begin{aligned}
\left(I+F+\ldots+F^{p q-1}\right) & =\left(I+F^{p}+\ldots+F^{p q-p}\right)\left(I+F+\ldots+F^{p-1}\right) \\
& =\left(I+F^{q}+\ldots+F^{p q-q}\right)\left(I+F+\ldots+F^{q-1}\right),
\end{aligned}
$$

implies that the right-hand side is contained in the left-hand side.

For the reverse inclusion, choose any element $\mathbf{x}$ in the left-hand side. Then, for some $\mathbf{y}, \mathbf{z} \in \mathbb{Z}^{2}$,

$$
\mathbf{x}=\left(I+F^{p}+\ldots+F^{p q-p}\right) \mathbf{y}, \quad \mathbf{x}=\left(I+F^{q}+\ldots+F^{p q-q}\right) \mathbf{z} .
$$

Thus

$$
\begin{aligned}
\left(I+F+\ldots+F^{p-1}\right) \mathbf{x} & =\left(I+F+\ldots+F^{p-1}\right)\left(I+F^{p}+\ldots+F^{p q-p}\right) \mathbf{y} \\
& =\left(I+F+\ldots+F^{p q-1}\right) \mathbf{y} \in \operatorname{Im}\left(I+F+\ldots+F^{p q-1}\right) .
\end{aligned}
$$


Similarly, we have

$$
\left(I+F+\ldots+F^{q-1}\right) \mathbf{x} \in \operatorname{Im}\left(I+F+\ldots+F^{p q-1}\right) .
$$

Since $\operatorname{Im}\left(I+F+\ldots+F^{p q-1}\right)$ is a subgroup of $\mathbb{Z}^{2}$ and is invariant under $F^{k}$ for all non-negative integer $k$. We may assume that $p<q$. Write

$$
q=\ell p+r, \quad 0<r<p, \quad(\ell, r \in \mathbb{Z}) .
$$

Then $\operatorname{gcd}(p, r)=1$ and

$$
\begin{aligned}
& \left(I+F+\ldots+F^{r-1}\right) \mathbf{x} \\
& =\left(I+F+\ldots+F^{q-1}\right) \mathbf{x}-\left(F^{r}+F^{r+p}+\ldots+F^{q-p}\right)\left(I+F+\ldots+F^{p-1}\right) \mathbf{x} \\
& \quad \in \operatorname{Im}\left(I+F+\ldots+F^{p q-1}\right) .
\end{aligned}
$$

Similarly, writing $p=\ell^{\prime} r+r^{\prime}, 0<r^{\prime}<r,\left(\ell^{\prime}, r^{\prime} \in \mathbb{Z}\right)$, we have

$$
\operatorname{gcd}\left(r, r^{\prime}\right)=1, \quad\left(I+F+\ldots+F^{r^{\prime}-1}\right) \mathbf{x} \in \operatorname{Im}\left(I+F+\ldots+F^{p q-1}\right) .
$$

Continuing this process, we can show that $\mathbf{x} \in \operatorname{Im}\left(I+F+\ldots+F^{p q-1}\right)$ as it is required, and hence completes the proof.

COROLlary 6.3. If $F$ is any integer square matrix and $p, q$ are relatively prime numbers, then

(a) $\operatorname{Im}\left(I+F^{p m}+\ldots+F^{p q m-p m}\right) \cap \operatorname{Im}\left(I+F^{q m}+\ldots+F^{p q m-q m}\right)$

$$
=\operatorname{Im}\left(I+F^{m}+\ldots+F^{p q m-m}\right) \text {. }
$$

(b) $\operatorname{Im}\left(I-F^{p m}+F^{2 p m}-\ldots+(-1)^{q-1} F^{(q-1) p m}\right) \cap \operatorname{Im}\left(I-F^{q m}+F^{2 q m}-\right.$ $\left.\ldots+(-1)^{q-1} F^{(p-1) q m}\right)=\operatorname{Im}\left(I-F^{m}+F^{2 m}-\ldots+(-1)^{p q-1} F^{(p q-1) m}\right)$.

(c) $1+x+\ldots+x^{p q-1}$ is the least common multiple of $1+x^{p}+\ldots+x^{(q-1) p}$ and $1+x^{q}+\ldots+x^{(p-1) q}$.

Proof. We obtain (a) and(b) by replacing $F$ by $F^{m}$ and $-F^{m}$, respectively, in Lemma 6.2.

When $F=[x]$ is an integer $1 \times 1$ matrix, $\operatorname{Im}\left(I+F^{p}+\ldots+F^{p q-p}\right)$ is the set of all multiples of $1+x^{p}+\ldots+x^{p q-p}$. Thus we can get (c) by Lemma 6.2.

LEMMA 6.4. If $n=2 p m$ where $p$ is an odd prime number, then $\operatorname{Im}\left(I-F^{p m}\right) \cap \operatorname{Im}\left(I+F^{2 m}+\ldots+F^{n-2 m}\right)=\operatorname{Im}\left(\left(I-F^{m}\right)\left(I+F^{2 m}+\ldots+F^{n-2 m}\right)\right)$. In particular, $\left(1-x^{2 p m}\right) /\left(1+x^{m}\right)=\left(1-x^{m}\right)\left(1+x^{2 m}+\ldots+x^{(p-1) 2 m}\right)$ is the least common multiple of $1-x^{p m}$ and $1+x^{2 m}+\ldots+x^{(p-1) 2 m}$.

Proof. Note that

$$
\begin{aligned}
I-F^{p m} & =\left(I-F^{m}\right)\left(I+F^{m}+\ldots+F^{p m-m}\right), \\
I+F^{2 m}+\ldots+F^{2 p m-2 m} & =\left(I-F^{m}+\ldots+\mid ! F^{p m-m}\right)\left(I+F^{m}+\ldots+F^{p m-m}\right) .
\end{aligned}
$$


Thus the right-hand side is contained in the left-hand side. Write the right-hand side by

$$
\begin{aligned}
Z & =\operatorname{Im}\left(\left(I-F^{m}\right)\left(I+F^{2 m}+\ldots+F^{n-2 m}\right)\right) \\
& =\operatorname{Im}\left(\left(I-F^{2 m}\right)\left(I-F^{m}+F^{2 m}-\ldots+F^{p m-m}\right)\right) .
\end{aligned}
$$

Choose any element $\mathbf{x}$ in the left-hand side. Then for some $\mathbf{y}, \mathbf{z} \in \mathbb{Z}^{2}$

$$
\mathbf{x}=\left(I-F^{p m}\right) \mathbf{y}, \quad \mathbf{x}=\left(I+F^{2 m}+\ldots+F^{n-2 m}\right) \mathbf{z} .
$$

Hence

$$
\left(I-F^{m}+\ldots+F^{p m-m}\right) \mathbf{x} \in Z, \quad\left(I-F^{m}\right) \mathbf{x} \in Z .
$$

Since the group $Z$ is invariant under $F^{k}$ for all non-negative integer $k$,

$\mathbf{x}=\left(I-F^{m}+\ldots+F^{p m-m}\right) \mathbf{x}+F^{m}\left(I+F^{2 m}+\ldots+F^{(p-3) m}\right)\left(I-F^{m}\right) \mathbf{x} \in Z$.

Hence the left-hand side is contained in the right-hand side.

THEOREM 6.5. Let $f$ be a self-map on $K$ of type $(F, \mathbf{b},-1)$. If $n=p_{1}^{e_{1}} \ldots p_{t}^{e_{t}}$ is odd and $\operatorname{det}\left(I+F^{n}\right)=0$ but $\operatorname{det}\left(I-F^{n}\right) \neq 0$, then the prime Nielsen-Jiang periodic number of period $n$ is

$$
N \mathrm{P}_{n}(f)=\left|\operatorname{det}\left(I-F^{n}\right)\right|+\sum_{\left\{k_{1}, \ldots, k_{s}\right\} \subset\{1, \ldots, t\}}(-1)^{s}\left|\operatorname{det}\left(I-F^{\left(n_{k_{1}}, \ldots, n_{k_{s}}\right)}\right)\right|,
$$

where the summation runs through the family of nonempty subsets $\left\{k_{1}, \ldots, k_{s}\right\}$ of $\{1, \ldots, t\}$.

Proof. Suppose that $\operatorname{det}\left(I-F^{n}\right) \neq 0$ and $\operatorname{det}\left(I+F^{n}\right)=0$. It is Remark 5.7 that

$$
\mathcal{R}\left[\varphi^{n}\right]=\left\{\left[s^{\overline{\mathbf{i}}}\right]_{n} \mid \overline{\mathbf{i}} \in \mathbb{Z}^{2} / \operatorname{Im}\left(I-F^{n}\right)\right\} \cup\left\{\left[s^{\overline{\mathbf{i}}} \alpha\right]_{n} \mid \overline{\mathbf{i}} \in \mathbb{Z}^{2} / \operatorname{Im}\left(I+F^{n}\right)\right\}
$$

where the first set is finite but the second set is infinite. Note also that for $m \mid n$, $I-F^{m}$ is a factor of $I-F^{n}$ and hence if $\operatorname{det}\left(I-F^{n}\right) \neq 0$ then $\operatorname{det}\left(I-F^{m}\right) \neq 0$. But one cannot expect that if $\operatorname{det}\left(I+F^{n}\right)=0$ then $\operatorname{det}\left(I+F^{m}\right)=0$.

We will show first that $\left\{\left[s^{\overline{\mathbf{i}}}\right]_{n} \mid, \overline{\mathbf{i}} \in \mathbb{Z}^{2} /\left(I-F^{n}\right)\left(\mathbb{Z}^{2}\right)\right\}$ is the set of all essential classes. The Reidemeister class $\left[s^{\overline{\mathbf{i}}}\right]_{n}$ corresponds to the fixed point class $p\left(\operatorname{Fix}\left(s^{\mathbf{i}} \widetilde{f}^{n}\right)\right)$ of $f^{n}$. Recalling from Lemma 4.6 that

$$
\tilde{f}=(\delta, D)=\left(\left[\begin{array}{c}
\mathbf{b} / 2 \\
*
\end{array}\right],\left[\begin{array}{cc}
F & 0 \\
0 & \omega
\end{array}\right]\right)=\left(\left[\begin{array}{c}
\mathbf{b} / 2 \\
*
\end{array}\right],\left[\begin{array}{rr}
F & 0 \\
0 & -1
\end{array}\right]\right)
$$

we have

$$
s^{\mathbf{i}} \tilde{f}^{n}=\left(\left[\begin{array}{l}
\mathbf{i} \\
0
\end{array}\right]+\left[\begin{array}{c}
\mathbf{b}_{n} / 2 \\
*
\end{array}\right],\left[\begin{array}{cr}
F^{n} & 0 \\
0 & -1
\end{array}\right]\right) .
$$

By a simple computation we see that $\operatorname{Fix}\left(s^{\mathbf{i}} \widetilde{f}^{n}\right)$ and hence $p\left(\operatorname{Fix}\left(s^{\mathbf{i}} \tilde{f}^{n}\right)\right)$ consist of a single element. This tells that the correspondence Reidemeister class $\left[s^{\overline{\mathbf{i}}}\right]_{n}$ 
is essential. Since $N\left(f^{n}\right)=\left|\operatorname{det}\left(I-F^{n}\right)\right|$, it follows that all other classes are inessential.

Next we will observe which are irreducible among the essential classes $\left[s^{\overline{\mathbf{i}}}\right]_{n}$. Suppose $\left[s^{\mathbf{i}}\right]_{n}$ is reducible to the level $m$. Then $\iota_{m, n}\left(\left[s^{\mathbf{x}} \alpha^{y}\right]_{m}\right)=\left[s^{\mathbf{i}}\right]_{n}$ for some $\left[s^{\mathbf{x}} \alpha^{y}\right]_{m} \in \mathcal{R}\left[\varphi^{m}\right]$. So $m \mid n$ and $y=0$. Note that as $n$ is odd, both $m$ and $n / m$ are odd.

Now a calculation shows that

$$
\iota_{m, n}\left(\left[s^{\mathbf{x}}\right]_{m}\right)=\left[s^{\mathbf{i}} \varphi^{m}\left(s^{\mathbf{x}}\right) \varphi^{2 m}\left(s^{\mathbf{x}}\right) \ldots \varphi^{n-m}\left(s^{\mathbf{x}}\right)\right]_{n}=\left[s^{\mathbf{x}_{m, n}}\right]_{n}
$$

where $\mathbf{x}_{m, n}=\left(I+F^{m}+F^{2 m}+\ldots+F^{n-m}\right) \mathbf{x}$. If $\mathbf{x} \in \operatorname{Im}\left(I-F^{m}\right)$ then clearly $\mathbf{x}_{m, n} \in \operatorname{Im}\left(I-F^{n}\right)$; if $\mathbf{x}_{m, n} \in \operatorname{Im}\left(I-F^{n}\right)$ then $\mathbf{x} \in \operatorname{Im}\left(I-F^{m}\right)$. For if $\mathbf{x}_{m, n}=\left(I-F^{n}\right) \mathbf{j}$ for some $\mathbf{j}$ then $\left(I+F^{m}+F^{2 m}+\ldots+F^{n-m}\right)\left(\left(I-F^{m}\right) \mathbf{j}-\mathbf{x}\right)=\mathbf{0}$ and as $I-F^{n}$ is invertible it follows that $\left(I-F^{m}\right) \mathbf{j}-\mathbf{x}=\mathbf{0}$. This observation shows that the homomorphism defined by multiplication by $I+F^{m}+\ldots+F^{n-m}$ is an isomorphism

(6.1) $\mathbf{x} \in \mathbb{Z}^{2} / \operatorname{Im}\left(I-F^{m}\right)$

$$
\mapsto \mathbf{x}_{m, n} \in \operatorname{Im}\left(I+F^{m}+F^{2 m}+\ldots+F^{n-m}\right) / \operatorname{Im}\left(I-F^{n}\right)
$$

and that the essential class $\left[s^{\overline{\mathbf{i}}}\right]_{n}$ is reducible to $m$ if and only if

$$
\overline{\mathbf{i}} \in \operatorname{Im}\left(I+F^{m}+\ldots+F^{n-m}\right) / \operatorname{Im}\left(I-F^{n}\right) \subset \mathbb{Z}^{2} / \operatorname{Im}\left(I-F^{n}\right) .
$$

Obviously, when $n=1$ all the essential classes $\left[s^{\overline{\mathbf{i}}}\right]_{1}$ are irreducible. Furthermore, for $n>1$ if $m\left|m^{\prime}\right| n$ and the essential class $\left[s^{\overline{1}}\right]_{n}$ is reducible to $m$, then it is reducible to $m^{\prime}$. Consequently, the essential class $\left[s^{\overline{\mathbf{i}}}\right]_{n}$ is reducible if and only if for some proper maximal divisor $m$ of $n$

$$
\overline{\mathbf{i}} \in C_{i}:=\operatorname{Im}\left(I+F^{m}+\ldots+F^{n-m}\right) / \operatorname{Im}\left(I-F^{n}\right) .
$$

Thus the set of essential irreducible classes is a one-to-one correspondence with the set

$$
\mathbb{Z}^{2} / \operatorname{Im}\left(I-F^{n}\right)-\bigcup_{j=1}^{t} C_{i} .
$$

By (6.1), $\# C_{i}=\left|\operatorname{det}\left(I-F^{\left(n_{i}\right)}\right)\right|$. For $1 \leq i<i^{\prime} \leq t$, by Corollary 6.3(a),

$$
C_{i} \cap C_{i^{\prime}}=\operatorname{Im}\left(I+F^{\left(n_{i}, n_{i^{\prime}}\right)}+\ldots+F^{n-\left(n_{i}, n_{i^{\prime}}\right)}\right) / \operatorname{Im}\left(I-F^{n}\right)
$$

and so, by (6.1) again, $\#\left(C_{i} \cap C_{i^{\prime}}\right)=\left|\operatorname{det}\left(I-F^{\left(n_{i}, n_{i^{\prime}}\right)}\right)\right|$. Using induction, we have

$$
\#\left(\bigcap_{i=1}^{s} C_{k_{i}}\right)=\left|\operatorname{det}\left(I-F^{\left(n_{k_{1}}, \ldots, n_{k_{s}}\right)}\right)\right| .
$$


Consequently,

$$
\#\left(\bigcup_{i=1}^{t} C_{i}\right)=\sum_{\left\{k_{1}, \ldots, k_{s}\right\} \subset\{1, \ldots, t\}}(-1)^{s-1}\left|\operatorname{det}\left(I-F^{\left(n_{k_{1}}, \ldots, n_{k_{s}}\right)}\right)\right| .
$$

Finally, we will find the length of each $\varphi$-orbit of the essential irreducible classes $\left[s^{\overline{\mathbf{i}}}\right]_{n}$. By definition,

$$
\left\langle\left[s^{\overline{\mathbf{i}}}\right]_{n}\right\rangle=\left\{\left[s^{\overline{\mathbf{i}}}\right]_{n},[\varphi]\left(\left[s^{\overline{\mathbf{i}}}\right]_{n}\right), \ldots,[\varphi]^{\ell-1}\left(\left[s^{\overline{\mathbf{i}}}\right]_{n}\right)\right\}=\left\{\left[s^{\overline{\mathbf{i}}}\right]_{n},\left[s^{F \overline{\mathbf{i}}}\right]_{n}, \ldots,\left[s^{F^{\ell-1} \overline{\mathbf{i}}}\right]_{n}\right\},
$$

where $\ell=\ell\left(\left[s^{\overline{\mathbf{i}}}\right]_{n}\right)$ is the length of $\left[s^{\overline{\mathbf{i}}}\right]_{n}$. Then $\ell \mid n$ and $\left[s^{\overline{\mathbf{i}}}\right]_{n}=\left[s^{F^{\ell \overline{\mathbf{i}}}}\right]_{n}$. This implies that $\left(I-F^{\ell}\right) \mathbf{i}=\left(I-F^{n}\right) \mathbf{j}$ for some $\mathbf{j} \in \mathbb{Z}^{2}$. So, $\left(I+F^{\ell}+\ldots+F^{n-\ell}\right) \mathbf{j}=\mathbf{i}$ or $\overline{\mathbf{i}} \in \operatorname{Im}\left(I+F^{\ell}+\ldots+F^{n-\ell}\right) / \operatorname{Im}\left(I-F^{n}\right)$. Thus this shows that all the irreducible essential Reidemeister classes have the same length $n$.

In all, we obtain that

$$
\begin{aligned}
O_{n}(\varphi) & =\frac{1}{n} \#\left(\mathbb{Z}^{2} / \operatorname{Im}\left(I-F^{n}\right)-\bigcup_{i=1}^{t} C_{i}\right) \\
& =\frac{1}{n}\left(\#\left(\mathbb{Z}^{2} / \operatorname{Im}\left(I-F^{n}\right)\right)-\#\left(\bigcup_{i=1}^{t} C_{i}\right)\right) \\
& =\frac{1}{n}\left(\left|\operatorname{det}\left(I-F^{n}\right)\right|+\sum_{\left\{k_{1}, \ldots, k_{s}\right\} \subset\{1, \ldots, t\}}(-1)^{s} \mid \operatorname{det}\left(I-F^{\left(n_{k_{1}}, \ldots, n_{k_{s}}\right)} \mid\right)\right.
\end{aligned}
$$

The conclusion now follows from the definition: $N \mathrm{P}_{n}(f)=n \times O_{n}(\varphi)$.

THEOREM 6.6. Let $f$ be a self-map on $K$ of type $(F, \mathbf{b},-1)$. If $n=p_{1}^{e_{1}} \ldots p_{t}^{e_{t}}$ is odd and $\operatorname{det}\left(I-F^{n}\right)=0$ but $\operatorname{det}\left(I+F^{n}\right) \neq 0$, then the prime Nielsen-Jiang periodic number of period $n$ is

$$
N \mathrm{P}_{n}(f)=\left|\operatorname{det}\left(I+F^{n}\right)\right|+\sum_{\left\{k_{1}, \ldots, k_{s}\right\} \subset\{1, \ldots, t\}}(-1)^{s}\left|\operatorname{det}\left(I+F^{\left(n_{k_{1}}, \ldots, n_{k_{s}}\right)}\right)\right|,
$$

where the summation runs through the family of nonempty subsets $\left\{k_{1}, \ldots, k_{s}\right\}$ of $\{1, \ldots, t\}$.

Proof. Let $f$ be a self-map on $K$ of type $(F, \mathbf{b},-1)$. Suppose that $n$ is odd and $\operatorname{det}\left(I-F^{n}\right)=0$ but $\operatorname{det}\left(I+F^{n}\right) \neq 0$. As it has been observed before, we may replace $F$ by $-F$. This means that $f$ is homotopic to a self-map on $K$ of type $(-F, \mathbf{b},-1)$, see Theorem 4.4. Since $n$ is odd, the conditions $\operatorname{det}\left(I-F^{n}\right)=0$ and $\operatorname{det}\left(I+F^{n}\right) \neq 0$ become $\operatorname{det}\left(I+(-F)^{n}\right)=0$ and $\operatorname{det}\left(I-(-F)^{n}\right) \neq 0$. Using the fact that the prime Nielsen periodic number is a homotopy invariant and applying Theorem 6.5, we have

$$
N \mathrm{P}_{n}(f)=\left|\operatorname{det}\left(I-(-F)^{n}\right)\right|+\sum_{\left\{k_{1}, \ldots, k_{s}\right\} \subset\{1, \ldots, t\}}(-1)^{s}\left|\operatorname{det}\left(I-(-F)^{\left(n_{k_{1}}, \ldots, n_{k_{s}}\right)}\right)\right| \text {. }
$$

This proves our result. 


\section{The Nielsen type numbers: non-weakly Jiang case II}

In this subsection we will discuss the remaining case where $\omega \neq \pm 1$ is odd and exactly one of $I-F^{n}$ and $I+F^{n}$ has zero determinant. Our computation problem of the Nielsen type numbers can be done in a similar, but much complicated way as we have done in the previous section by the following general four steps.

Step 1. Finding the Reidemeister classes $\mathcal{R}\left[\varphi^{n}\right]$.

By Remark 5.8, we have

$$
\begin{aligned}
\mathcal{R}\left[\varphi^{n}\right]= & \left\{\left[s^{\overline{\mathbf{i}}} \alpha^{j}\right]_{n}\left|\overline{\mathbf{i}} \in \mathbb{Z}^{2} / \operatorname{Im}\left(I-F^{n}\right) ; j=0,2, \ldots,\right| 1-\omega^{n} \mid-2\right\} \\
& \cup\left\{\left[s^{\overline{\mathbf{i}}} \alpha^{j}\right]_{n}\left|\overline{\mathbf{i}} \in \mathbb{Z}^{2} / \operatorname{Im}\left(I+F^{n}\right) ; j=1,3, \ldots,\right| 1-\omega^{n} \mid-1\right\} .
\end{aligned}
$$

Step 2. Finding the essential Reidemeister classes.

Recalling from Lemma 4.6 that

$$
\widetilde{f}=(\delta, D)=\left(\left[\begin{array}{c}
\mathbf{b} / 2 \\
*
\end{array}\right],\left[\begin{array}{cc}
F & 0 \\
0 & \omega
\end{array}\right]\right),
$$

we have

$$
s^{\mathbf{i}} \alpha^{j} \widetilde{f}^{n}=\left(\left[\begin{array}{c}
\mathbf{i} \\
j / 2
\end{array}\right]+\left[\begin{array}{c}
(-1)^{j} \mathbf{b}_{n} / 2 \\
*^{\prime}
\end{array}\right],\left[\begin{array}{cc}
(-1)^{j} F^{n} & 0 \\
0 & \omega^{n}
\end{array}\right]\right),
$$

where $*^{\prime}=\left(1+\omega+\ldots+\omega^{n-1}\right) *$. By a simple computation we see that $\operatorname{Fix}\left(s^{\mathbf{i}} \alpha^{j} \widetilde{f}^{n}\right)$ is:

when $j$ is even,

$$
\operatorname{Fix}\left(s^{\mathbf{i}} \alpha^{j} \widetilde{f}^{n}\right)=\left\{\left[\begin{array}{l}
\mathbf{x} \\
y
\end{array}\right] \mid\left(I-F^{n}\right) \mathbf{x}=\mathbf{i}+\frac{1}{2} \mathbf{b}_{n},\left(1-\omega^{n}\right) y=\frac{j}{2}+*^{\prime}\right\} ;
$$

when $j$ is odd,

$$
\operatorname{Fix}\left(s^{\mathbf{i}} \alpha^{j} \widetilde{f}^{n}\right)=\left\{\left[\begin{array}{c}
\mathbf{x} \\
y
\end{array}\right] \mid\left(I+F^{n}\right) \mathbf{x}=\mathbf{i}-\frac{1}{2} \mathbf{b}_{n},\left(1-\omega^{n}\right) y=\frac{j}{2}+*^{\prime}\right\} .
$$

We consider first the case that $\operatorname{det}\left(I-F^{n}\right) \neq 0$ and $\operatorname{det}\left(I+F^{n}\right)=0$. When $j$ is even $\left.\operatorname{Fix}\left(s^{\mathbf{i}} \alpha^{j} \widetilde{f}^{n}\right)\right)$ and hence $p\left(\operatorname{Fix}\left(s^{\mathbf{i}} \alpha^{j} \widetilde{f}^{n}\right)\right)$ consist of a single element. This tells that the correspondence Reidemeister classes $\left[s^{\overline{\mathrm{i}}} \alpha^{j}\right]_{n}$ are essential. Since $N\left(f^{n}\right)=\left|1-\omega^{n}\right|\left|\operatorname{det}\left(I-F^{n}\right)\right| / 2$, it follows that all other classes are inessential. Thus the essential classes are

$$
E C_{1}:=\left\{\left[s^{\overline{\mathbf{i}}} \alpha^{j}\right]_{n}\left|\overline{\mathbf{i}} \in \mathbb{Z}^{2} / \operatorname{Im}\left(I-F^{n}\right) ; j=0,2, \ldots,\right| 1-\omega^{n} \mid-2\right\} .
$$

If the other case occurs, in a similar way we can see that the essential classes are

$$
E C_{2}:=\left\{\left[s^{\overline{\mathbf{i}}} \alpha^{j}\right]_{n}\left|\overline{\mathbf{i}} \in \mathbb{Z}^{2} / \operatorname{Im}\left(I+F^{n}\right) ; j=1,3, \ldots,\right| 1-\omega^{n} \mid-1\right\} .
$$


Before moving to the next steps, we recall that

$$
\varphi^{k}\left(s^{\mathbf{x}} \alpha^{y}\right)= \begin{cases}s^{F^{k} \mathbf{x}} \alpha^{\omega^{k} y} & \text { when } y \text { is even; } \\ s^{F^{k} \mathbf{x}+\mathbf{b}_{k}} \alpha^{\omega^{k} y} & \text { when } y \text { is odd }\end{cases}
$$

Let $m \mid n$. When $y$ is even,

$$
\begin{aligned}
\iota_{m, n}\left(\left[s^{\mathbf{x}} \alpha^{y}\right]_{m}\right) & =\left[\left(s^{\mathbf{x}} \alpha^{y}\right) \varphi^{m}\left(s^{\mathbf{x}} \alpha^{y}\right) \varphi^{2 m}\left(s^{\mathbf{x}} \alpha^{y}\right) \ldots \varphi^{n-m}\left(s^{\mathbf{x}} \alpha^{y}\right)\right]_{n} \\
& =\left[\left(s^{\mathbf{x}} \alpha^{y}\right)\left(s^{F^{m}} \mathbf{x} \alpha^{\omega^{m} y}\right)\left(s^{F^{2 m}} \mathbf{x} \alpha^{\omega^{2 m} y}\right) \ldots\left(s^{F^{n-m} \mathbf{x}} \alpha^{\omega^{n-m} y}\right)\right]_{n} \\
& =\left[s^{\left(I+F^{m}+F^{2 m}+\ldots+F^{n-m}\right) \mathbf{x}} \alpha^{\left(1+\omega^{m}+\omega^{2 m}+\ldots+\omega^{n-m}\right) y}\right]_{n} \\
& :=\left[s^{\mathbf{x}_{m, n}^{\prime}} \alpha^{y_{m, n}}\right]_{n} ;
\end{aligned}
$$

when $y$ is odd,

$$
\begin{aligned}
& \iota_{m, n}\left(\left[s^{\mathbf{x}} \alpha^{y}\right]_{m}\right) \\
& =\left[\left(s^{\mathbf{x}} \alpha^{y}\right) \varphi^{m}\left(s^{\mathbf{x}} \alpha^{y}\right) \varphi^{2 m}\left(s^{\mathbf{x}} \alpha^{y}\right) \ldots \varphi^{n-m}\left(s^{\mathbf{x}} \alpha^{y}\right)\right]_{n} \\
& =\left[\left(s^{\mathbf{x}} \alpha^{y}\right)\left(s^{F^{m} \mathbf{x}+\mathbf{b}_{m}} \alpha^{\omega^{m} y}\right)\left(s^{F^{2 m} \mathbf{x}+\mathbf{b}_{2 m}} \alpha^{\omega^{2 m} y}\right) \ldots\left(s^{F^{n-m} \mathbf{x}+\mathbf{b}_{n-m}} \alpha^{\omega^{n-m} y}\right)\right]_{n} \\
& :=\left[s^{\mathbf{x}_{m, n}^{\prime \prime}+\mathbf{b}_{m, n}} \alpha^{y_{m, n}}\right]_{n},
\end{aligned}
$$

where

$$
\begin{aligned}
& \mathbf{x}_{m, n}^{\prime}=\left(I+F^{m}+F^{2 m}+\ldots+F^{n-m}\right) \mathbf{x}, \\
& y_{m, n}=\left(1+\omega^{m}+\omega^{2 m}+\ldots+\omega^{n-m}\right) y, \\
& \mathbf{x}_{m, n}^{\prime \prime}=\left(I-F^{m}+\ldots+(-1)^{n / m-1} F^{n-m}\right) \mathbf{x}, \\
& \mathbf{b}_{m, n}=-\mathbf{b}_{m}+\mathbf{b}_{2 m}-\ldots+(-1)^{n / m-1} \mathbf{b}_{n-m} .
\end{aligned}
$$

Note here that $1+\omega^{m}+\ldots+\omega^{n-m}$ is an odd integer if $n / m$ is odd; otherwise it is an even integer.

LEMMA 7.1. If $n=p q m$ where $m$ is even and $p, q$ are relatively prime odd numbers, then

$$
\begin{aligned}
&\left(\mathbf{b}_{p m, n}+\operatorname{Im}\left(I-F^{p m}+F^{2 p m}\right.\right.\left.\left.-\ldots+F^{n-p m}\right)\right) \\
& \cap\left(\mathbf{b}_{q m, m}+\operatorname{Im}\left(I-F^{q m}+F^{2 q m}-\ldots+F^{n-q m}\right)\right) \\
& \quad=\mathbf{b}_{m, n}+\operatorname{Im}\left(I-F^{m}+F^{2 m}-\ldots+F^{n-m}\right) .
\end{aligned}
$$

Proof. By a simple computation, we can show that

$$
I-F^{m}+\ldots+F^{n-m}=\left(I-F^{p m}+\ldots+F^{n-p m}\right)\left(I-F^{m}+\ldots+F^{p m-m}\right),
$$


and

$$
\begin{aligned}
\mathbf{b}_{m, n}= & \left(-\mathbf{b}_{m}+\mathbf{b}_{2 m}\right)+\ldots+\left(-\mathbf{b}_{(p-2) m}+\mathbf{b}_{(p-1) m}\right)-\mathbf{b}_{p m} \\
& +\left(\mathbf{b}_{(p+1) m}-\mathbf{b}_{(p+2) m}\right)+\ldots \\
& +\left(\mathbf{b}_{(2 p-2) m}-\mathbf{b}_{(2 p-1) m}\right)+\mathbf{b}_{2 p m}+\ldots \\
& +\left(\mathbf{b}_{((q-2) p+1) m}-\mathbf{b}_{((q-2) p+2) m}\right)+\ldots \\
& +\left(\mathbf{b}_{((q-1) p-2) m}-\mathbf{b}_{((q-1) p-1) m}\right)+\mathbf{b}_{n-p m} \\
& +\left(-\mathbf{b}_{((q-1) p+1) m}+\mathbf{b}_{((q-1) p+2) m}\right)+\ldots+\left(-\mathbf{b}_{(p q-2) m}+\mathbf{b}_{(p q-1) m}\right) \\
= & \mathbf{b}_{p m, n}+\left(I-F^{p m}+\ldots+F^{n-p m}\right) F^{m}\left(I+F^{2 m}+\ldots+F^{(p-3) m}\right) \mathbf{b}_{m} .
\end{aligned}
$$

This implies that

$$
\mathbf{b}_{m, n}-\mathbf{b}_{p m, n} \in \operatorname{Im}\left(I-F^{p m}+\ldots+F^{n-p m}\right) .
$$

A similar computation yields that

$$
\mathbf{b}_{m, n}-\mathbf{b}_{q m, n} \in \operatorname{Im}\left(I-F^{q m}+\ldots+F^{n-q m}\right) .
$$

Now it follows that the right-hand side is contained in the left-hand side.

For the reverse inclusion, we assume

$$
\begin{aligned}
& \mathbf{i}-\mathbf{b}_{p m, n} \in \operatorname{Im}\left(I-F^{p m}+\ldots+F^{n-p m}\right), \\
& \mathbf{i}-\mathbf{b}_{q m, n} \in \operatorname{Im}\left(I-F^{q m}+\ldots+F^{n-q m}\right) .
\end{aligned}
$$

By (7.1) and (7.2), we have

$$
\mathbf{i}-\mathbf{b}_{m, n} \in \operatorname{Im}\left(I-F^{q m}+\ldots+F^{n-q m}\right) \cap \operatorname{Im}\left(I-F^{p m}+\ldots+F^{n-p m}\right) .
$$

By Corollary 6.3-(2),

$$
\begin{aligned}
\mathbf{i}-\mathbf{b}_{m, n} & \in \operatorname{Im}\left(I-F^{p m}+\ldots+F^{n-p m}\right) \cap \operatorname{Im}\left(I-F^{q m}+\ldots+F^{n-q m}\right) \\
& =\operatorname{Im}\left(I-F^{m}+\ldots+F^{n-m}\right) .
\end{aligned}
$$

This shows the reverse inclusion.

Lemma 7.2. Let $n=2^{e_{0}} p_{1}^{e_{1}} \ldots p_{t}^{e_{t}}$ be even. Let

$$
\begin{aligned}
& J_{0}^{\prime}=\left\{j|0 \leq j<| 1-\omega^{n} \mid, j=(2 k+1)\left(1+\omega^{\left(n_{0}\right)}\right) \text { for some } k\right\}, \\
& J_{i}=\left\{j|0 \leq j<| 1-\omega^{n} \mid, j=2 \ell\left(1+\omega^{\left(n_{i}\right)}+\ldots+\omega^{n-\left(n_{i}\right)}\right) \text { for some } \ell\right\} .
\end{aligned}
$$

For each $i=1, \ldots, t$, we have

$$
\begin{aligned}
& J_{0}^{\prime} \cap J_{i}=\left\{c\left|1+\omega^{\left(n_{0}, n_{i}\right)}+\ldots+\omega^{n-\left(n_{0}, n_{i}\right)}\right| \mid\right. \\
& \left.\quad c \text { is odd with } 0<c<\left|1-\omega^{\left(n_{0}, n_{i}\right)}\right|\right\} .
\end{aligned}
$$

In particular, $J_{0}^{\prime} \cap J_{i}$ has $\left|1-\omega^{\left(n_{0}, n_{i}\right)}\right| / 2$ elements. 
Proof. Fix $i \in\{1, \ldots, t\}$, and let $p=p_{i}, n=2 p m$ and $x=\omega^{m}=\omega^{\left(n_{0}, n_{i}\right)}$. By Lemma 6.4,

$$
\begin{aligned}
\operatorname{gcd}\left(1+x^{p}, 1+x^{2}+\ldots+x^{2(p-1)}\right)=\frac{\left(1+x^{p}\right)\left(1+x^{2}+\ldots+x^{2(p-1)}\right)}{1+x+x^{2}+\ldots+x^{2 p-1}} \\
=\frac{1+x^{2}+\ldots+x^{2(p-1)}}{1+x+x^{2}+\ldots+x^{p-1}}=1-x+x^{2}-\ldots+x^{p-1} .
\end{aligned}
$$

Thus

$$
\begin{aligned}
(2 k+1)\left|1+x^{p}\right|=2 \ell\left|1+x^{2}+\ldots+x^{2(p-1)}\right|, & \\
\text { with } 0 & \leq 2 k+1<\left|1-x^{p}\right| \text { and } 0 \leq 2 \ell<\left|1-x^{2}\right|
\end{aligned}
$$

turns into

$$
(2 k+1)|1+x|=2 \ell\left|1+x+x^{2}+\ldots+x^{p-1}\right| .
$$

Since $\operatorname{gcd}\left(1+x, 1+x+x^{2}+\ldots+x^{p-1}\right)=1$, we must have

$$
2 k+1=c\left|1+x+x^{2}+\ldots+x^{p-1}\right|, \quad 2 \ell=c|1+x|
$$

for some odd $c$ with $0<c<|1-x|$. This finishes the proof.

COROLlaRY 7.3. The set $J_{0}^{\prime} \cap\left(\bigcap_{i=1}^{s} J_{k_{i}}\right)$ has $\left|1-\omega^{\left(n_{0}, n_{k_{1}}, \ldots, n_{k_{s}}\right)}\right| / 2$ elements.

Proof. By Corollary 6.3, $j \in \bigcap_{i=1}^{s} J_{k_{i}}$ if and only if $0 \leq j<\left|1-\omega^{n}\right|$ and $j$ is a multiple of $2\left(1+\omega^{\left(n_{k_{1}}, \ldots, n_{k_{s}}\right)}+\ldots+\omega^{n-\left(n_{k_{1}}, \ldots, n_{k_{s}}\right)}\right)$. Next, we simply apply Lemma 7.2 and finish the proof.

Now, according as $n$ is odd or even we will consider the next two steps.

7.1. When $n$ is odd. For any $m$ with $m \mid n$, both $m$ and $n / m$ are odd.

CASE 1. $\operatorname{det}\left(I-F^{n}\right) \neq 0$ and $\operatorname{det}\left(I+F^{n}\right)=0$.

In this case, we will consider the next two steps in a row.

Step 3. Finding the irreducible essential Reidemeister classes.

The essential classes are

$$
E C:=\left\{\left[s^{\overline{\mathbf{i}}} \alpha^{j}\right]_{n}\left|\overline{\mathbf{i}} \in \mathbb{Z}^{2} / \operatorname{Im}\left(I-F^{n}\right) ; j=0,2, \ldots,\right| 1-\omega^{n} \mid-2\right\}
$$

whose cardinality is $\left|\operatorname{det}\left(I-F^{n}\right)\right| \times\left|1-\omega^{n}\right| / 2$. Suppose $\left[s^{\overline{\mathbf{i}}} \alpha^{j}\right]_{n}$ is reducible to the level $m$. Then $\left[s^{\mathbf{i}} \alpha^{j}\right]_{n}=\iota_{m, n}\left(\left[s^{\mathbf{x}} \alpha^{y}\right]_{m}\right)=\left[s^{\mathbf{x}_{m, n}^{\prime}} \alpha^{y_{m, n}}\right]_{n}$ for some $\left[s^{\mathbf{x}} \alpha^{y}\right]_{m} \in$ $\mathcal{R}\left[\varphi^{m}\right]$. Because $n$ is odd and $j$ is even, as we observed above, $y_{m, n}$ is even if and only if $y$ is even. This tells that we only need to consider the essential Reidemeister classes of the form $\left[s^{\mathbf{x}} \alpha^{y}\right]_{m}$ where $y$ is even and $\overline{\mathbf{i}} \in \mathbb{Z}^{2} / \operatorname{Im}\left(I-F^{m}\right)$ under the boost function $\iota_{m, n}$.

We observe that $\mathbf{x} \in \operatorname{Im}\left(I-F^{m}\right)$ if and only if $\mathbf{x}_{m, n}^{\prime} \in \operatorname{Im}\left(I-F^{n}\right)$ as before (see the proof of Theorem 6.5). Moreover, if $0 \leq y<\left|1-\omega^{m}\right|$ then 
$0 \leq y_{m, n}<\left|1-\omega^{n}\right|$, and the converse holds. This observation gives rise to an one-one correspondence between

$$
\mathbb{Z}^{2} / \operatorname{Im}\left(I-F^{m}\right) \times\left\{y \mid y \text { even, } 0 \leq y<\left|1-\omega^{m}\right|\right\}
$$

and

$$
\begin{aligned}
\operatorname{Im}\left(I+F^{m}+\ldots+F^{n-m}\right) / \operatorname{Im}\left(I-F^{n}\right) & \\
& \times\left\{j \mid j \text { is a multiple of } 2\left(1+\omega^{m}+\ldots+\omega^{n-m}\right), 0 \leq j<\left|1-\omega^{n}\right|\right\} .
\end{aligned}
$$

Furthermore, if $m\left|m^{\prime}\right| n$ and the essential class $\left[s^{\overline{\mathbf{i}}} \alpha^{j}\right]_{n}$ is reducible to $m$, then the essential class $\left[s^{\overline{\mathrm{i}}} \alpha^{j}\right]_{n}$ is reducible to $m^{\prime}$. Consequently, the essential class $\left[s^{\overline{\mathrm{i}}} \alpha^{j}\right]_{n}$ is reducible if and only if for some $i \in\{1, \ldots, t\}$,

- $\overline{\mathbf{i}} \in \operatorname{Im}\left(I+F^{n_{i}}+\ldots+F^{n-n_{i}}\right) / \operatorname{Im}\left(I-F^{n}\right)$,

- $j$ is a multiple of $2\left(1+\omega^{n_{i}}+\ldots+\omega^{n-n_{i}}\right)$.

For each $i=1, \ldots, t$, let

$$
\begin{array}{r}
C_{i}=\left\{\left[s^{\overline{\mathrm{i}}} \alpha^{j}\right]_{n} \in O C \mid \overline{\mathbf{i}} \in \operatorname{Im}\left(I+F^{\left(n_{i}\right)}+\ldots+F^{n-\left(n_{i}\right)}\right) / \operatorname{Im}\left(I-F^{n}\right) ;\right. \\
\left.j \text { is a multiple of } 2\left(1+\omega^{\left(n_{i}\right)}+\ldots+\omega^{n-\left(n_{i}\right)}\right)\right\} .
\end{array}
$$

Then

$$
\# C_{i}=\left|\operatorname{det}\left(I-F^{\left(n_{i}\right)}\right)\right| \times \frac{1}{2}\left|1-\omega^{\left(n_{i}\right)}\right|
$$

and for $1 \leq i<i^{\prime} \leq t$, we have that $\left[s^{\overline{\mathrm{i}}} \alpha^{j}\right]_{n} \in C_{i} \cap C_{i^{\prime}}$ if and only if

- $\overline{\mathbf{i}} \in \operatorname{Im}\left(I+F^{\left(n_{i}, n_{i^{\prime}}\right)}+\ldots+F^{n-\left(n_{i}, n_{i^{\prime}}\right)}\right) / \operatorname{Im}\left(I-F^{n}\right)$,

- $j$ is a multiple of $2\left(1+\omega^{\left(n_{i}, n_{i^{\prime}}\right)}+\ldots+\omega^{n-\left(n_{i}, n_{i^{\prime}}\right)}\right)$.

Here, the conditions above follow from Corollary 6.3(a) and (c). This shows that

$$
\#\left(C_{i} \cap C_{i^{\prime}}\right)=\left|\operatorname{det}\left(I-F^{\left(n_{i}, n_{i^{\prime}}\right)}\right)\right| \times \frac{1}{2}\left|1-\omega^{\left(n_{i}, n_{i^{\prime}}\right)}\right| .
$$

By induction, we have

$$
\#\left(\bigcap_{i=1}^{s} C_{k_{i}}\right)=\left|\operatorname{det}\left(I-F^{\left(n_{k_{1}}, \ldots, n_{k_{s}}\right)}\right)\right| \times \frac{1}{2}\left|1-\omega^{\left(n_{k_{1}}, \ldots, n_{k_{s}}\right)}\right| .
$$

Thus

$$
\#\left(\bigcup_{i=1}^{t} C_{i}\right)=\sum_{\left\{k_{1}, \ldots, k_{s}\right\} \subset\{1, \ldots, t\}}(-1)^{s-1} \mid \operatorname{det}\left(I-F^{\left(n_{k_{1}}, \ldots, n_{k_{s}}\right)}\left|\times \frac{1}{2}\right| 1-\omega^{\left(n_{k_{1}}, \ldots, n_{k_{s}}\right)} \mid .\right.
$$

Step 4. Finding the length of irreducible essential Reidemeister classes.

Let $\left[s^{\overline{\mathbf{i}}} \alpha^{j}\right]_{n}$ be an irreducible essential Reidemeister class. By definition,

$$
\begin{aligned}
\left\langle\left[s^{\overline{\mathbf{i}}} \alpha^{j}\right]_{n}\right\rangle & =\left\{\left[s^{\overline{\mathbf{i}}} \alpha^{j}\right]_{n},[\varphi]\left(\left[s^{\overline{\mathbf{i}}} \alpha^{j}\right]_{n}\right), \ldots,[\varphi]^{\ell-1}\left(\left[s^{\overline{\mathbf{i}}} \alpha^{j}\right]_{n}\right)\right\} \\
= & \begin{cases}\left.\left\{s^{\overline{\mathbf{i}}} \alpha^{j}\right]_{n},\left[s^{F \overline{\mathbf{i}}} \alpha^{\omega j}\right]_{n}, \ldots,\left[s^{F^{\ell-1}} \overline{\mathbf{i}} \alpha^{\omega^{\ell-1} j}\right]_{n}\right\} & \text { when } j \text { is even; } \\
\left\{\left[s^{\mathbf{i}} \alpha^{j}\right]_{n},\left[s^{F \overline{\mathbf{i}}+\mathbf{b}_{1}} \alpha^{\omega j}\right]_{n}, \ldots,\left[s^{F^{\ell-1} \overline{\mathbf{i}}+\mathbf{b}_{\ell-1}} \alpha^{\omega^{\ell-1} j}\right]_{n}\right\} & \text { when } j \text { is odd, }\end{cases}
\end{aligned}
$$


where $\ell=\ell\left(\left[s^{\overline{\mathrm{i}}} \alpha^{j}\right]_{n}\right)$ is the length of $\left[s^{\overline{\mathrm{i}}} \alpha^{j}\right]_{n}$. Then $\ell \mid n$ and

$$
\left[s^{\overline{\mathbf{i}}}\right]_{n}=[\varphi]^{\ell}\left(\left[s^{\overline{\mathbf{i}}} \alpha^{j}\right]_{n}\right)= \begin{cases}{\left[s^{F^{\ell} \overline{\mathbf{i}}} \alpha^{\omega^{\ell} j}\right]_{n}} & \text { when } j \text { is even; } \\ {\left[s^{F^{\ell \overline{\mathbf{i}}+\mathbf{b}_{\ell}} \alpha^{\omega^{\ell} j}}\right]_{n}} & \text { when } j \text { is odd. }\end{cases}
$$

Since $j$ is even, $\left[s^{\overline{\mathbf{i}}} \alpha^{j}\right]_{n}=\left[s^{F^{\ell} \overline{\mathbf{i}}} \alpha^{\omega^{\ell} j}\right]_{n}$. This implies that

$$
s^{F^{\ell} \mathbf{i}} \alpha^{\omega^{\ell} j}=\left(s^{\mathbf{x}} \alpha^{y}\right)\left(s^{\mathbf{i}} \alpha^{j}\right) \varphi^{n}\left(s^{\mathbf{x}} \alpha^{y}\right)^{-1}
$$

for some $s^{\mathbf{x}} \alpha^{y} \in \Pi$. By Remark 5.8, this identity turns into

$$
s^{F^{\ell} \mathbf{i}} \alpha^{\omega^{\ell} j}= \begin{cases}s^{-\mathbf{i}+\left(I-F^{n}\right) \mathbf{x}-\mathbf{b}_{n}} \alpha^{y\left(1-\omega^{n}\right)+j} & \text { when } y \text { is odd; } \\ s^{\mathbf{i}+\left(I-F^{n}\right) \mathbf{x}} \alpha^{y\left(1-\omega^{n}\right)+j} & \text { when } y \text { is even. }\end{cases}
$$

Since $\left(1-\omega^{\ell}\right) j=\left(1-\omega^{n}\right)(-y)$, we have $j=\left(1+\omega^{\ell}+\ldots+\omega^{n-\ell}\right)(-y)$. As $n$ is odd $1+\omega^{\ell}+\ldots+\omega^{n-\ell}$ is odd; hence as $j$ is even $y$ must be even. As a result, $\left(I-F^{\ell}\right) \mathbf{i}=\left(I-F^{n}\right)(-\mathbf{x}),\left(I+F^{\ell}+\ldots+F^{n-\ell}\right)(-\mathbf{x})=\mathbf{i}$ or $\overline{\mathbf{i}} \in$ $\operatorname{Im}\left(I+F^{\ell}+\ldots+F^{n-\ell}\right) / \operatorname{Im}\left(I-F^{n}\right)$. Thus this shows that the essential class $\left[s^{\overline{\mathrm{i}}} \alpha^{j}\right]_{n}$ is reducible to $\ell$. Hence if it is irreducible its length must be $n$. That is, all the irreducible essential Reidemeister classes have the same length $n$.

In all,

$$
\begin{aligned}
N \mathrm{P}_{n}(f)= & \#\left(E C-\bigcup_{i=1}^{t} C_{i}\right)=\#(E C)-\#\left(\bigcup_{i=1}^{t} C_{i}\right) \\
= & \frac{1}{2}\left(\left|\operatorname{det}\left(I-F^{n}\right)\right| \times\left|1-\omega^{n}\right|\right. \\
& +\sum_{\left\{k_{1}, \ldots, k_{s}\right\} \subset\{1, \ldots, t\}}(-1)^{s} \mid \operatorname{det}\left(I-F^{\left(n_{k_{1}}, \ldots, n_{k_{s}}\right)}|\times| 1-\omega^{\left(n_{k_{1}}, \ldots, n_{k_{s}}\right)} \mid\right) .
\end{aligned}
$$

CASE 2. $\operatorname{det}\left(I-F^{n}\right)=0$ and $\operatorname{det}\left(I+F^{n}\right) \neq 0$.

Recall that $f$ is homotopic to a self-map on $K$ of type $(-F, \mathbf{a}, \omega)$. Thus in this case it holds that $\operatorname{det}\left(I-(-F)^{n}\right) \neq 0$ and $\operatorname{det}\left(I+(-F)^{n}\right)=0$. Applying the above case for $-F$, we can deduce the following:

- The essential classes are

$$
\left\{\left[s^{\overline{\mathbf{i}}} \alpha^{j}\right]_{n}\left|\overline{\mathbf{i}} \in \mathbb{Z}^{2} / \operatorname{Im}\left(I-(-F)^{n}\right) ; j=0,2 \ldots,\right| 1-\omega^{n} \mid-2\right\} .
$$

- The essential class $\left[s^{\overline{\mathrm{i}}} \alpha^{j}\right]_{n}$ is reducible if and only if for some $i \in\{1, \ldots, t\}$,

(a) $\overline{\mathbf{i}} \in \operatorname{Im}\left(I+(-F)^{n_{i}}+\ldots+(-F)^{n-n_{i}}\right) / \operatorname{Im}\left(I-(-F)^{n}\right)$,

(b) $j$ is a multiple of $2\left(1+\omega^{n_{i}}+\ldots+\omega^{n-n_{i}}\right)$.

- $N \mathrm{P}_{n}(f)=\frac{1}{2}\left(\left|\operatorname{det}\left(I-(-F)^{n}\right)\right| \times\left|1-\omega^{n}\right|\right.$

$$
+\sum_{\left\{k_{1}, \ldots, k_{s}\right\} \subset\{1, \ldots, t\}}(-1)^{s} \mid \operatorname{det}\left(I-(-F)^{\left(n_{k_{1}}, \ldots, n_{k_{s}}\right)}|\times| 1-\omega^{\left(n_{k_{1}}, \ldots, n_{k_{s}}\right)} \mid\right)
$$




$$
\begin{aligned}
= & \frac{1}{2}\left(\left|\operatorname{det}\left(I+F^{n}\right)\right| \times\left|1-\omega^{n}\right|\right. \\
& +\sum_{\left\{k_{1}, \ldots, k_{s}\right\} \subset\{1, \ldots, t\}}(-1)^{s} \mid \operatorname{det}\left(I+F^{\left(n_{k_{1}}, \ldots, n_{k_{s}}\right)}|\times| 1-\omega^{\left(n_{k_{1}}, \ldots, n_{k_{s}}\right)} \mid\right) .
\end{aligned}
$$

7.2. When $n$ is even. In this subsection, we will analyze Step 3 first and then Step 4 in a row.

Step 3. Finding the irreducible essential Reidemeister classes.

Case 1. $\operatorname{det}\left(I-F^{n}\right) \neq 0$ and $\operatorname{det}\left(I+F^{n}\right)=0$.

The essential classes are

$$
E C_{1}:=\left\{\left[s^{\overline{\mathbf{i}}} \alpha^{j}\right]_{n}\left|\overline{\mathbf{i}} \in \mathbb{Z}^{2} / \operatorname{Im}\left(I-F^{n}\right) ; j=0,2, \ldots,\right| 1-\omega^{n} \mid-2\right\}
$$

whose cardinality is $\left|\operatorname{det}\left(I-F^{n}\right)\right| \times\left|1-\omega^{n}\right| / 2$. Let $n=2^{e_{0}} p_{1}^{e_{1}} \ldots p_{t}^{e_{t}}$ be the prime decomposition of the even integer $n$. We ascertain the following:

Claim. The essential class $\left[s^{\overline{\mathrm{i}}} \alpha^{j}\right]_{n}$ is reducible if and only if for some $i \in$ $\{0, \ldots, t\}$

- $\overline{\mathbf{i}} \in \operatorname{Im}\left(I+F^{\left(n_{i}\right)}+\ldots+F^{n-\left(n_{i}\right)}\right) / \operatorname{Im}\left(I-F^{n}\right)$,

- $j$ is a multiple of $2\left(1+\omega^{\left(n_{i}\right)}+\ldots+\omega^{n-\left(n_{i}\right)}\right)$

or

- $\overline{\mathbf{i}} \in-\overline{\mathbf{b}}_{\left(n_{0}\right)}+\operatorname{Im}\left(I-F^{\left(n_{0}\right)}\right) / \operatorname{Im}\left(I-F^{n}\right)$,

- $j-\left(1+\omega^{\left(n_{0}\right)}\right)$ is a multiple of $2\left(1+\omega^{\left(n_{0}\right)}\right)$.

We recall from Step 2 the following:

$$
\iota_{m, n}\left(\left[s^{\mathbf{x}} \alpha^{y}\right]_{m}\right)= \begin{cases}{\left[s^{\mathbf{x}_{m, n}^{\prime}} \alpha^{y_{m, n}}\right]_{n}} & \text { when } y \text { is even, } \\ {\left[s^{\mathbf{x}_{m, n}^{\prime \prime}+\mathbf{b}_{m, n}} \alpha^{y_{m, n}}\right]_{n}} & \text { when } y \text { is odd. }\end{cases}
$$

Notice that we are concerned with the case where $y_{m, n}=\left(1+\omega^{m}+\omega^{2 m}+\ldots+\right.$ $\left.\omega^{n-m}\right) y$ is even. In this case, we have either $y$ is even or $y$ is odd and $n / m$ is even.

Assume that $y$ is even. We note that $\mathbf{x} \in \operatorname{Im}\left(I-F^{m}\right)$ if and only if $\mathbf{x}_{m, n}^{\prime} \in$ $\operatorname{Im}\left(I-F^{n}\right)$ (see the proof of Theorem 6.5). Furthermore, if $0 \leq y<\left|1-\omega^{m}\right|$ then $0 \leq y_{m, n}<\left|1-\omega^{n}\right|$, and the converse holds. This observation gives rise to an one-one correspondence between

$$
\mathbb{Z}^{2} / \operatorname{Im}\left(I-F^{m}\right) \times\left\{y \mid y \text { even, } 0 \leq y<\left|1-\omega^{m}\right|\right\}
$$

and

$$
\begin{aligned}
\operatorname{Im}\left(I+F^{m}+\ldots+F^{n-m}\right) / \operatorname{Im}\left(I-F^{n}\right) & \\
& \times\left\{j \mid j \text { a multiple of } 2\left(1+\omega^{m}+\ldots+\omega^{n-m}\right), 0 \leq j<\left|1-\omega^{n}\right|\right\} .
\end{aligned}
$$

Furthermore, if $m\left|m^{\prime}\right| n$ and the essential class $\left[s^{\overline{\mathbf{i}}} \alpha^{j}\right]_{n}$ is reducible to $\left[s^{\mathbf{x}} \alpha^{y}\right] m$ where $y$ is even, then the essential class $\left[s^{\overline{\mathbf{i}}} \alpha^{j}\right]_{n}$ is reducible to $\left[s^{\mathbf{x}^{\prime}} \alpha^{y^{\prime}}\right]_{m^{\prime}}$ where 
$y^{\prime}$ is even. Consequently, the essential class $\left[s^{\overline{\mathbf{i}}} \alpha^{j}\right]_{n}$ is reduced to an (essential) Reidemeister class of the form $\left[s^{x} \alpha^{y}\right]_{m}$ where $y$ is even if and only if for some $i \in\{0, \ldots, t\}$

- $\overline{\mathbf{i}} \in \operatorname{Im}\left(I+F^{n_{i}}+\ldots+F^{n-n_{i}}\right) / \operatorname{Im}\left(I-F^{n}\right)$,

- $j$ is a multiple of $2\left(1+\omega^{n_{i}}+\ldots+\omega^{n-n_{i}}\right)$.

Assume that $n / m$ even. Then $I+F^{m}$ is a factor of $I-F^{n}$ and so $\operatorname{det}(I+$ $\left.F^{m}\right) \neq 0$. This implies that $N\left(f^{m}\right)=R\left(f^{m}\right)$ and so $f^{m}$ is weakly Jiang, even though $f^{n}$ is not weakly Jiang. Assume in addition that $y$ is odd and so $\overline{\mathbf{x}} \in \mathbb{Z}^{2} / \operatorname{Im}\left(I+F^{m}\right)$ (see Step 1). If there is $m^{\prime}<n$ such that $m\left|m^{\prime}\right| n$ and $m^{\prime} / m$ is even, then $\left(1+\omega^{m}+\ldots+\omega^{m^{\prime}-m}\right) y$ is even. Hence the boost function $\iota_{m, m^{\prime}}$ sends a Reidemeister class $\left[s^{\overline{\mathbf{x}}} \alpha^{y}\right]_{m}$ with $y$ odd to a Reidemeister class $\left[s^{\overline{\mathbf{x}}^{\prime}} \alpha^{y^{\prime}}\right]_{m^{\prime}}$ with $y^{\prime}$ even. Therefore this case turns into the case where $y$ is even, and it was treated in the above. Therefore we have to consider the case where if $m^{\prime}<n$ with $m\left|m^{\prime}\right| n$ then $\frac{m^{\prime}}{m}$ is odd. This happens only when $m=n / 2$. The correspondence $\mathbf{x} \mapsto \mathbf{x}_{m, n}^{\prime \prime}+\mathbf{b}_{m, n}=\left(I-F^{m}\right) \mathbf{x}-\mathbf{b}_{m}$ gives rise to an one-one correspondence

$$
\begin{aligned}
\mathbb{Z}^{2} / \operatorname{Im}\left(I+F^{m}\right) & \stackrel{\cong}{\longrightarrow} \operatorname{Im}\left(I-F^{m}\right) / \operatorname{Im}\left(I-F^{n}\right) \\
& \longleftrightarrow-\overline{\mathbf{b}}_{m}+\operatorname{Im}\left(I-F^{m}\right) / \operatorname{Im}\left(I-F^{n}\right) .
\end{aligned}
$$

Also, there is an one-one correspondence from $\left\{y \mid y\right.$ odd, $\left.0 \leq y<\left|1-\omega^{m}\right|\right\}$ onto

$$
\left\{j|j=| 1+\omega^{m} \mid(2 k+1), 0 \leq k<\frac{\left|1-\omega^{m}\right|}{2}\right\} .
$$

Remark that when $m=n / 2$, the boost function $\iota_{m, n}$ sends a Reidemeister class $\left[s^{\overline{\mathbf{x}}} \alpha^{y}\right]_{m}$ with $y$ even to a Reidemeister class $\left[s^{\overline{\mathbf{x}}^{\prime}} \alpha^{y^{\prime}}\right]_{n}$, where $y^{\prime}$ is a multiple of $2\left(1+\omega^{m}\right)$. This tells that any two Reidemeister classes $\left[s^{\overline{\mathbf{x}}_{1}} \alpha^{y_{1}}\right]_{m}$ and $\left[s^{\overline{\mathbf{x}}_{2}} \alpha^{y_{2}}\right]_{m}$ with $y_{1}$ even and $y_{2}$ odd are boosted to two distinct Reidemeister classes in level $n$. Hence the essential class $\left[s^{\overline{\mathrm{i}}} \alpha^{j}\right]_{n}$ is reducible to $m=\left(n_{0}\right)=n / 2$ if and only if either

- $\overline{\mathbf{i}} \in \operatorname{Im}\left(I+F^{\left(n_{0}\right)}\right) / \operatorname{Im}\left(I-F^{n}\right)$,

- $j$ is a multiple of $2\left(1+\omega^{\left(n_{0}\right)}\right)$

or

- $\overline{\mathbf{i}} \in-\overline{\mathbf{b}}_{\left(n_{0}\right)}+\operatorname{Im}\left(I-F^{\left(n_{0}\right)}\right) / \operatorname{Im}\left(I-F^{n}\right)$,

- $j-\left(1+\omega^{\left(n_{0}\right)}\right)$ is a multiple of $2\left(1+\omega^{\left(n_{0}\right)}\right)$.

This completes the proof of our claim.

Now let

$$
\begin{aligned}
C_{0}^{\prime}=\left\{\left[s^{\overline{\mathbf{i}}} \alpha^{j}\right]_{n} \in E C_{1} \mid \overline{\mathbf{i}} \in-\overline{\mathbf{b}}_{\left(n_{0}\right)}+\operatorname{Im}\left(I-F^{\left(n_{0}\right)}\right) / \operatorname{Im}\left(I-F^{n}\right) ;\right. \\
\left.j \text { a multiple of } 2\left(1+\omega^{\left(n_{0}\right)}\right) \text { plus }\left|1+\omega^{\left(n_{0}\right)}\right|\right\},
\end{aligned}
$$


and let, for each $i=0, \ldots, t$,

$$
\begin{array}{r}
C_{i}=\left\{\left[s^{\overline{\mathrm{i}}} \alpha^{j}\right]_{n} \in E C_{1} \mid \overline{\mathbf{i}} \in \operatorname{Im}\left(I+F^{\left(n_{i}\right)}+\ldots+F^{n-\left(n_{i}\right)}\right) / \operatorname{Im}\left(I-F^{n}\right) ;\right. \\
\left.j \text { a multiple of } 2\left(1+\omega^{\left(n_{i}\right)}+\ldots+\omega^{n-\left(n_{i}\right)}\right)\right\} .
\end{array}
$$

Then

$$
\begin{aligned}
& \# C_{0}^{\prime}=\left|\operatorname{det}\left(I+F^{\left(n_{0}\right)}\right)\right| \times \frac{1}{2}\left|1-\omega^{\left(n_{0}\right)}\right|, \\
& \# C_{i}=\left|\operatorname{det}\left(I-F^{\left(n_{i}\right)}\right)\right| \times \frac{1}{2}\left|1-\omega^{\left(n_{i}\right)}\right| .
\end{aligned}
$$

As it was observed before,

$$
\#\left(C_{0} \cap C_{0}^{\prime}\right)=0, \quad \#\left(\bigcap_{i=1}^{s} C_{k_{i}}\right)=\left|\operatorname{det}\left(I-F^{\left(n_{k_{1}}, \ldots, n_{k_{s}}\right)}\right)\right| \times \frac{1}{2}\left|1-\omega^{\left(n_{k_{1}}, \ldots, n_{k_{s}}\right)}\right| .
$$

Now to determine $C_{0}^{\prime} \cap C_{i}$, first we claim that

$$
\left(-\mathbf{b}_{\left(n_{0}\right)}+\operatorname{Im}\left(I-F^{\left(n_{0}\right)}\right)\right) \cap \operatorname{Im}\left(I+F^{\left(n_{i}\right)}+\ldots+F^{n-\left(n_{i}\right)}\right) \neq \emptyset .
$$

This is equivalent to show that there exist $\mathbf{x}, \mathbf{y}$ such that

$$
-\left(I+F+\ldots+F^{\left(n_{0}\right)-1}\right) \mathbf{b}+\left(I-F^{\left(n_{0}\right)}\right) \mathbf{x}=\left(I+F^{\left(n_{i}\right)}+\ldots+F^{n-\left(n_{i}\right)}\right) \mathbf{y} .
$$

Multiplying the inverse of $I+F^{\left(n_{0}, n_{i}\right)}+\ldots+F^{\left(n_{0}\right)-\left(n_{0}, n_{i}\right)}$ on both sides, we obtain

$$
\begin{gathered}
-\left(I+F+\ldots+F^{\left(n_{0}, n_{i}\right)-1}\right) \mathbf{b}+\left(I-F^{\left(n_{0}, n_{i}\right)}\right) \mathbf{x}\left[=\left(I-F^{\left(n_{i}\right)}+\ldots+F^{n-\left(n_{i}\right)}\right) \mathbf{y}\right] \\
=\mathbf{y}-\left(I-F^{\left(n_{0}, n_{i}\right)}\right)\left(F^{\left(n_{0}, n_{i}\right)}+F^{3\left(n_{0}, n_{i}\right)}+\ldots+F^{\left(n_{0}\right)-\left(n_{i}\right)}\right) \mathbf{y} .
\end{gathered}
$$

Thus we can choose $\mathbf{x}, \mathbf{y}$, in fact we choose

$$
\begin{aligned}
& \mathbf{y}=-\left(I+F+\ldots+F^{\left(n_{0}, n_{i}\right)-1}\right) \mathbf{b}, \\
& \mathbf{x}=-\left(F^{\left(n_{0}, n_{i}\right)}+F^{3\left(n_{0}, n_{i}\right)}+\ldots+F^{\left(n_{0}\right)-\left(n_{i}\right)}\right) \mathbf{y}
\end{aligned}
$$

so that the above equality holds. Now we fix $\mathbf{i}_{0}$ such that

$$
\mathbf{i}_{0} \in\left(-\mathbf{b}_{\left(n_{0}\right)}+\operatorname{Im}\left(I-F^{\left(n_{0}\right)}\right)\right) \cap\left(\operatorname{Im}\left(I+F^{\left(n_{i}\right)}+\ldots+F^{n-\left(n_{i}\right)}\right)\right) .
$$

Then it can be seen easily that

$$
\begin{aligned}
\left(-\mathbf{b}_{n_{0}}+\operatorname{Im}\left(I-F^{n_{0}}\right)\right) \cap & \left(\operatorname{Im}\left(I+F^{n_{i}}+\ldots+F^{n-n_{i}}\right)\right) \\
& =\mathbf{i}_{0}+\left(\operatorname{Im}\left(I-F^{n_{0}}\right) \cap \operatorname{Im}\left(I+F^{n_{i}}+\ldots+F^{n-n_{i}}\right)\right) .
\end{aligned}
$$

By Lemma 6.4, we have

$$
\begin{aligned}
& \operatorname{Im}\left(I-F^{n_{0}}\right) / \operatorname{Im}\left(I-F^{n}\right) \cap \operatorname{Im}\left(I+F^{n_{i}}+\ldots+F^{n-n_{i}}\right) / \operatorname{Im}\left(I-F^{n}\right) \\
& \quad=\operatorname{Im}\left(\left(I-F^{\left(n_{0}, n_{i}\right)}\right)\left(I+F^{n_{i}}+\ldots+F^{n-n_{i}}\right)\right) / \operatorname{Im}\left(I-F^{n}\right) \\
& \quad \cong \mathbb{Z}^{2} / \operatorname{Im}\left(I+F^{\left(n_{0}, n_{i}\right)}\right) .
\end{aligned}
$$


By Corollary 6.4 and 7.3, we can conclude that

$$
\#\left(C_{0}^{\prime} \cap C_{i}\right)=\left|\operatorname{det}\left(I+F^{\left(n_{0}, n_{i}\right.}\right)\right| \times \frac{1}{2}\left|1-\omega^{\left(n_{0}, n_{i}\right)}\right| .
$$

By induction, we have

$$
\#\left(\bigcap_{i=1}^{u}\left(C_{0}^{\prime} \cap C_{k_{i}}\right)\right)=\mid \operatorname{det}\left(I+F^{\left(n_{0}, n_{k_{1}}, \ldots, n_{k_{u}}\right)}\left|\times \frac{1}{2}\right| 1-\omega^{\left(n_{0}, n_{k_{1}}, \ldots, n_{k_{u}}\right)} \mid .\right.
$$

Consequently, we have

$$
\begin{aligned}
& \#\left(C_{0}^{\prime} \cup\left(\bigcup_{i=0}^{t} C_{i}\right)\right)=\# C_{0}^{\prime}+\#\left(\bigcup_{i=0}^{t} C_{i}\right)-\#\left(C_{0}^{\prime} \cap\left(\bigcup_{i=0}^{t} C_{i}\right)\right) \\
&= \# C_{0}^{\prime}+\#\left(\bigcup_{i=0}^{t} C_{i}\right)-\#\left(\bigcup_{i=1}^{t}\left(C_{0}^{\prime} \cap C_{i}\right)\right) \\
&= \frac{1}{2}\left|\operatorname{det}\left(I+F^{n_{0}}\right)\right| \times\left|1-\omega^{n_{0}}\right| \\
&-\frac{1}{2} \sum_{\left\{k_{1}, \ldots, k_{s}\right\} \subset\{0, \ldots, t\}}(-1)^{s}\left|\operatorname{det}\left(I-F^{\left(n_{k_{1}}, \ldots, n_{k_{s}}\right)}\right)\right| \times\left|1-\omega^{\left(n_{k_{1}}, \ldots, n_{k_{s}}\right)}\right| \\
&+\frac{1}{2} \sum_{\left\{k_{1}, \ldots, k_{u}\right\} \subset\{1, \ldots, t\}}(-1)^{u}\left|\operatorname{det}\left(I+F^{\left(n_{0}, n_{k_{1}}, \ldots, n_{k_{u}}\right)}\right)\right| \times\left|1-\omega^{\left(n_{0}, n_{k_{1}}, \ldots, n_{k_{u}}\right)}\right| .
\end{aligned}
$$

CAse 2. $\operatorname{det}\left(I-F^{n}\right)=0$ and $\operatorname{det}\left(I+F^{n}\right) \neq 0$.

The essential classes are

$$
E C_{2}:=\left\{\left[s^{\overline{\mathbf{i}}} \alpha^{j}\right]_{n}\left|\overline{\mathbf{i}} \in \mathbb{Z}^{2} / \operatorname{Im}\left(I+F^{n}\right) ; j=1,3, \ldots,\right| 1-\omega^{n} \mid-1\right\}
$$

whose cardinality is $\left|\operatorname{det}\left(I+F^{n}\right)\right| \times\left|1-\omega^{n}\right| / 2$. Recall again from Step 2 that

$$
\iota_{m, n}\left(\left[s^{\mathbf{x}} \alpha^{y}\right]_{m}\right)= \begin{cases}{\left[s^{\mathbf{x}_{m, n}^{\prime}} \alpha^{y_{m, n}}\right]_{n}} & \text { when } y \text { is even; } \\ {\left[s^{\mathbf{x}_{m, n}^{\prime \prime}+\mathbf{b}_{m, n}} \alpha^{y_{m, n}}\right]_{n}} & \text { when } y \text { is odd }\end{cases}
$$

where $y_{m, n}=\left(1+\omega^{m}+\ldots+\omega^{n-m}\right) y$ is odd. So, we must have both $y$ and $n / m$ are odd. Thus we need to observe the identity

$$
\iota_{m, n}\left(\left[s^{\mathbf{x}} \alpha^{y}\right]_{m}\right)=\left[s^{\mathbf{x}_{m, n}^{\prime \prime}+\mathbf{b}_{m, n}} \alpha^{y_{m, n}}\right]_{n} .
$$

We observe that $\mathbf{x} \in \operatorname{Im}\left(I+F^{m}\right)$ if and only if $\mathbf{x}_{m, n}^{\prime \prime} \in \operatorname{Im}\left(I+F^{n}\right)$ as before. And if $0 \leq y<\left|1-\omega^{m}\right|$ then $0 \leq y_{m, n}<\left|1-\omega^{n}\right|$, and the converse holds. Furthermore, if $m\left|m^{\prime}\right| n$ and the essential class $\left[s^{\overline{\mathbf{i}}} \alpha^{j}\right]_{n}$ is reducible to $m$, then $\left[s^{\overline{\mathbf{i}}} \alpha^{j}\right]_{n}$ is reducible to $m^{\prime}$. This observation implies that the essential class $\left[s^{\overline{\mathbf{i}}} \alpha^{j}\right]_{n}$ is reducible if and only if for some proper maximal divisor $m$ of $n$ with $n / m$ odd,

- $\overline{\mathbf{i}}-\overline{\mathbf{b}}_{m, n} \in \operatorname{Im}\left(I-F^{m}+\ldots+F^{n-m}\right) / \operatorname{Im}\left(I+F^{n}\right)$,

- $j$ is odd and is multiple of $\left(1+\omega^{m}+\ldots+\omega^{n-m}\right)$. 
For each $i=1, \ldots, t$, let

$$
C_{i}=\left\{(\overline{\mathbf{i}}, j) \in E C_{2} \mid \overline{\mathbf{i}}-\overline{\mathbf{b}}_{n_{i}, n} \in \operatorname{Im}\left(I-F^{n_{i}}+\ldots+F^{n-n_{i}}\right) / \operatorname{Im}\left(I+F^{n}\right),\right.
$$
$j$ is odd and a multiple of $\left.\left(1+\omega^{n_{i}}+\ldots+\omega^{n-n_{i}}\right)\right\}$.

Then

$$
\# C_{i}=\left|\operatorname{det}\left(I+F^{n_{i}}\right)\right| \times \frac{1}{2}\left|1-\omega^{n_{i}}\right| .
$$

By Lemma 7.1 and Corollary 6.3 , we have that $\left[s^{\overline{\mathbf{i}}} \alpha^{j}\right]_{n} \in \bigcap_{i=1}^{u} C_{k_{i}}$ if and only if - $\overline{\mathbf{i}}-\overline{\mathbf{b}}_{\left(n_{k_{1}}, \ldots, n_{k_{u}}\right), n} \in \operatorname{Im}\left(I-F^{\left(n_{k_{1}}, \ldots, n_{k_{u}}\right)}+\ldots+F^{n-\left(n_{k_{1}}, \ldots, n_{k_{u}}\right)}\right) / \operatorname{Im}\left(I-F^{n}\right)$,

- $j$ is odd and a multiple of $1+\omega^{\left(n_{k_{1}}, \ldots, n_{k_{u}}\right)}+\ldots+\omega^{n-\left(n_{k_{1}}, \ldots, n_{k_{u}}\right)}$.

Thus we can conclude that

$$
\#\left(\bigcap_{i=1}^{u} C_{k_{i}}\right)=\mid \operatorname{det}\left(I+F^{\left(n_{k_{1}}, \ldots, n_{k_{u}}\right)}\left|\times \frac{1}{2}\right| 1-\omega^{\left(n_{k_{1}}, \ldots, n_{k_{u}}\right)} \mid .\right.
$$

Consequently, we have

$\#\left(\bigcup_{i=1}^{t} C_{i}\right)=\frac{1}{2} \sum_{\left\{k_{1}, \ldots, k_{s}\right\} \subset\{1, \ldots, t\}}(-1)^{s-1}\left|\operatorname{det}\left(I+F^{\left(n_{k_{1}}, \ldots, n_{k_{s}}\right)}\right)\right| \times\left|1-\omega^{\left(n_{k_{1}}, \ldots, n_{k_{s}}\right)}\right|$.

Step 4. Finding the length of irreducible essential Reidemeister classes.

Let $\left[s^{\overline{\mathbf{i}}} \alpha^{j}\right]_{n}$ be an irreducible essential Reidemeister class. By definition,

$$
\begin{aligned}
& \left\langle\left[s^{\overline{\mathbf{i}}} \alpha^{j}\right]_{n}\right\rangle=\left\{\left[s^{\overline{\mathbf{i}}} \alpha^{j}\right]_{n},[\varphi]\left(\left[s^{\overline{\mathbf{i}}} \alpha^{j}\right]_{n}\right), \ldots,[\varphi]^{\ell-1}\left(\left[s^{\overline{\mathbf{i}}} \alpha^{j}\right]_{n}\right)\right\} \\
& \quad= \begin{cases}\left\{\left[s^{\overline{\mathbf{i}}} \alpha^{j}\right]_{n},\left[s^{F \overline{\mathbf{i}}} \alpha^{\omega j}\right]_{n}, \ldots,\left[s^{F^{\ell-1}} \overline{\mathbf{i}} \alpha^{\omega^{\ell-1}} j\right]_{n}\right\} & \text { when } j \text { is even; } \\
\left\{\left[s^{\overline{\mathbf{i}}} \alpha^{j}\right]_{n},\left[s^{F \overline{\mathbf{i}}+\mathbf{b}_{1}} \alpha^{\omega j}\right]_{n}, \ldots,\left[s^{F^{\ell-1} \overline{\mathbf{i}}+\mathbf{b}_{\ell-1}} \alpha^{\omega^{\ell-1} j}\right]_{n}\right\} & \text { when } j \text { is odd, }\end{cases}
\end{aligned}
$$

where $\ell=\ell\left(\left[s^{\overline{\mathbf{i}}} \alpha^{j}\right]_{n}\right)$ is the length of $\left[s^{\overline{\mathbf{i}}} \alpha^{j}\right]_{n}$. Then $\ell \mid n$ and

$$
\left[s^{\overline{\mathbf{i}}}\right]_{n}=[\varphi]^{\ell}\left(\left[s^{\overline{\mathbf{i}}} \alpha^{j}\right]_{n}\right)= \begin{cases}{\left[s^{F^{\ell} \overline{\mathbf{i}}} \alpha^{\omega^{\ell} j}\right]_{n}} & \text { when } j \text { is even; } \\ {\left[s^{F^{\ell} \overline{\mathbf{i}}+\mathbf{b}_{\ell}} \alpha^{\omega^{\ell} j}\right]_{n}} & \text { when } j \text { is odd }\end{cases}
$$

CAsE 3. $\operatorname{det}\left(I-F^{n}\right) \neq 0$ and $\operatorname{det}\left(I+F^{n}\right)=0$.

In this case $j$ must be even. Thus $\left[s^{\overline{\mathbf{i}}} \alpha^{j}\right]_{n}=\left[s^{F^{\ell} \overline{\mathbf{i}}} \alpha^{\omega^{\ell} j}\right]_{n}$. This implies that

$$
s^{F^{\ell} \mathbf{i}} \alpha^{\omega^{\ell} j}=\left(s^{\mathbf{x}} \alpha^{y}\right)\left(s^{\mathbf{i}} \alpha^{j}\right) \varphi^{n}\left(s^{\mathbf{x}} \alpha^{y}\right)^{-1}
$$

for some $s^{\mathbf{x}} \alpha^{y} \in \Pi$. By Remark 5.8, this identity turns into

$$
s^{F^{\ell} \mathbf{i}} \alpha^{\omega^{\ell} j}= \begin{cases}s^{-\mathbf{i}+\left(I-F^{n}\right) \mathbf{x}-\mathbf{b}_{n}} \alpha^{y\left(1-\omega^{n}\right)+j} & \text { when } y \text { is odd } \\ s^{\mathbf{i}+\left(I-F^{n}\right) \mathbf{x}} \alpha^{y\left(1-\omega^{n}\right)+j} & \text { when } y \text { is even. }\end{cases}
$$


Since $\left(1-\omega^{\ell}\right) j=\left(1-\omega^{n}\right)(-y)$, we have $j=\left(1+\omega^{\ell}+\ldots+\omega^{n-\ell}\right)(-y)$. Thus either $n / \ell$ is even or $n / \ell$ is odd and $y$ is even.

Assume that $n / \ell$ is even. Then $\ell \mid(n / 2)$ and so we may assume that $\ell=n / 2$. By the above identity, we can see that

$$
\begin{cases}\left(I+F^{\ell}\right) \mathbf{i}=\left(I-F^{n}\right) \mathbf{x}-\mathbf{b}_{n}=\left(I+F^{\ell}\right)\left\{\left(I-F^{\ell}\right) \mathbf{x}-\mathbf{b}_{\ell}\right\} & \text { when } y \text { is odd; } \\ \left(I-F^{\ell}\right) \mathbf{i}=\left(I-F^{n}\right)(-\mathbf{x})=\left(I-F^{\ell}\right)\left(I+F^{\ell}\right)(-\mathbf{x}) & \text { when } y \text { is even. }\end{cases}
$$

This yields that

$$
\mathbf{i}= \begin{cases}\left(I-F^{\ell}\right) \mathbf{x}-\mathbf{b}_{\ell} & \text { when } y \text { is odd } \\ \left(I+F^{\ell}\right)(-\mathbf{x}) & \text { when } y \text { is even. }\end{cases}
$$

These tell that $\left[s^{\mathbf{i}} \alpha^{j}\right]_{n}$ is reducible to $\ell=n / 2$, a contradiction.

Assume that $n / \ell$ is odd and $y$ is even. The fact that $y$ is even implies that $\left(I-F^{\ell}\right) \mathbf{i}=\left(I-F^{n}\right)(-\mathbf{x}), \mathbf{i}=\left(I+F^{\ell}+\ldots+F^{n-\ell}\right)(-\mathbf{x})$ or $\overline{\mathbf{i}} \in \operatorname{Im}\left(I+F^{\ell}+\ldots\right.$ $\left.+F^{n-\ell}\right) / \operatorname{Im}\left(I-F^{n}\right)$. Thus this shows that the essential class $\left[s^{\overline{\mathbf{i}}} \alpha^{j}\right]_{n}$ is reducible to $\ell$. Hence as it is irreducible its length must be $n$.

In all, all the irreducible essential Reidemeister classes have the same length $n$. Therefore, we obtain that

$$
\begin{aligned}
N P_{n}(f)=\#\left(E C_{1}-\left(C_{0}^{\prime} \cup\left(\bigcup_{i=0}^{t} C_{i}\right)\right)\right) \\
=\#\left(E C_{1}\right)-\#\left(C_{0}^{\prime} \cup\left(\bigcup_{i=0}^{t} C_{i}\right)\right) \\
=\frac{1}{2}\left(\left|\operatorname{det}\left(I-F^{n}\right)\right| \times\left|1-\omega^{n}\right|-\left|\operatorname{det}\left(I+F^{n_{0}}\right)\right| \times\left|1-\omega^{n_{0}}\right|\right. \\
\quad+\sum_{\left\{k_{1}, \ldots, k_{s}\right\} \subset\{0,1, \ldots, t\}}(-1)^{s}\left|\operatorname{det}\left(I-F^{\left(n_{k_{1}}, \ldots, n_{k_{s}}\right)}\right)\right| \times\left|1-\omega^{\left(n_{k_{1}}, \ldots, n_{k_{s}}\right)}\right| \\
\left.\quad-\sum_{\left\{k_{1}, \ldots, k_{u}\right\} \subset\{1, \ldots, t\}}(-1)^{u}\left|\operatorname{det}\left(I+F^{\left(n_{0}, n_{k_{1}}, \ldots, n_{k_{u}}\right)}\right)\right| \times\left|1-\omega^{\left(n_{0}, n_{k_{1}}, \ldots, n_{k_{u}}\right)}\right|\right) .
\end{aligned}
$$

CASE $4 . \operatorname{det}\left(I-F^{n}\right)=0$ and $\operatorname{det}\left(I+F^{n}\right) \neq 0$.

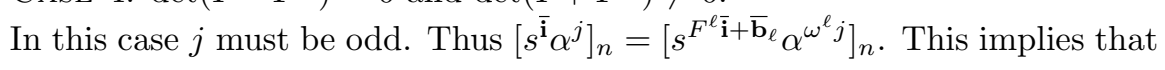

$$
s^{F^{\ell} \mathbf{i}+\mathbf{b}_{\ell}} \alpha^{\omega^{\ell} j}=\left(s^{\mathbf{x}} \alpha^{y}\right)\left(s^{\mathbf{i}} \alpha^{j}\right) \varphi^{n}\left(s^{\mathbf{x}} \alpha^{y}\right)^{-1}
$$

for some $s^{\mathrm{x}} \alpha^{y} \in \Pi_{1}$. By Remark 5.8, this identity turns into

$$
s^{F^{\ell} \mathbf{i}+\mathbf{b}_{\ell}} \alpha^{\omega^{\ell} j}= \begin{cases}s^{-\mathbf{i}+\left(I+F^{n}\right) \mathbf{x}+\mathbf{b}_{n}} \alpha^{y\left(1-\omega^{n}\right)+j} & \text { when } y \text { is odd; } \\ s^{\mathbf{i}+\left(I+F^{n}\right) \mathbf{x}} \alpha^{y\left(1-\omega^{n}\right)+j} & \text { when } y \text { is even. }\end{cases}
$$


This yields that $j=y\left(1+\omega^{\ell}+\ldots+\omega^{n-\ell}\right)$. As $j$ is odd, so are $y$ and $n / \ell$. Furthermore,

$$
\left(I+F^{\ell}\right) \mathbf{i}+\mathbf{b}_{\ell}-\mathbf{b}_{n}=\left(I+F^{n}\right) \mathbf{x}=\left(I+F^{\ell}\right)\left(I-F^{\ell}+\ldots+F^{n-\ell}\right) \mathbf{x}
$$

or

$$
\left(I+F^{\ell}\right) \mathbf{i}-\left(I+F^{\ell}\right) \mathbf{b}_{\ell, n}=\left(I+F^{\ell}\right)\left(I-F^{\ell}+\ldots+F^{n-\ell}\right) \mathbf{x} .
$$

Hence $\mathbf{i}-\mathbf{b}_{\ell, n} \in \operatorname{Im}\left(I-F^{\ell}+\ldots+F^{n-\ell}\right)$.

In all, the irreducible essential class $\left[s^{\overline{\mathrm{i}}} \alpha^{j}\right]_{n}$ is reducible to $\ell$ and so $\ell=n$. Thus all the irreducible essential Reidemeister classes have the same length $n$. Therefore, we obtain that

$$
\begin{aligned}
N \mathrm{P}_{n}(f)= & \#\left(E C_{2}-\bigcup_{i=1}^{t} C_{i}\right)=\#\left(E C_{2}\right)-\#\left(\bigcup_{i=1}^{t} C_{i}\right) \\
= & \frac{1}{2}\left(\left|\operatorname{det}\left(I+F^{n}\right)\right| \times\left|1-\omega^{n}\right|\right. \\
& \left.+\sum_{\left\{k_{1}, \ldots, k_{s}\right\} \subset\{1, \ldots, t\}}(-1)^{s}\left|\operatorname{det}\left(I+F^{\left(n_{k_{1}}, \ldots, n_{k_{s}}\right)}\right)\right| \times\left|1-\omega^{\left(n_{k_{1}}, \ldots, n_{k_{s}}\right)}\right|\right) .
\end{aligned}
$$

With these observation so far, we have:

THEOREM 7.4. Let $f$ be a self-map on $K$ of type $(F, \mathbf{b}, \omega)$. If $\omega \neq \pm 1$ is odd and $\operatorname{det}\left(I-F^{n}\right) \neq 0$ but $\operatorname{det}\left(I+F^{n}\right)=0$, then the prime Nielsen-Jiang periodic number of $f$ of period $n$ is given as follows:

(a) when $n=p_{1}^{e_{1}} \ldots p_{t}^{e_{t}}$ is odd,

$$
\begin{aligned}
N \mathrm{P}_{n}(f)= & \frac{1}{2}\left(\left|\operatorname{det}\left(I-F^{n}\right)\right|\left|1-\omega^{n}\right|\right. \\
& +\sum_{\left\{k_{1}, \ldots, k_{s}\right\} \subset\{1, \ldots, t\}}(-1)^{s} \mid \operatorname{det}\left(I-F^{\left(n_{k_{1}}, \ldots, n_{k_{s}}\right)}|| 1-\omega^{\left(n_{k_{1}}, \ldots, n_{k_{s}}\right)} \mid\right) ;
\end{aligned}
$$

(b) when $n=2^{e_{0}} p_{1}^{e_{1}} \ldots p_{t}^{e_{t}}$ is even,

$$
\begin{aligned}
N \mathrm{P}_{n}(f) & =\frac{1}{2}\left(\left|\operatorname{det}\left(I-F^{n}\right)\right|\left|1-\omega^{n}\right|-\left|\operatorname{det}\left(I+F^{\left(n_{0}\right)}\right)\right|\left|1-\omega^{\left(n_{0}\right)}\right|\right. \\
& +\sum_{\substack{\left\{k_{1}, \ldots, k_{s}\right\} \\
\subset\{0,1, \ldots, t\}}}(-1)^{s}\left|\operatorname{det}\left(I-F^{\left(n_{k_{1}}, \ldots, n_{k_{s}}\right)}\right)\right|\left|1-\omega^{\left(n_{k_{1}}, \ldots, n_{k_{s}}\right)}\right| \\
& \left.-\sum_{\substack{\left\{k_{1}, \ldots, k_{u}\right\} \\
\subset\{1, \ldots, t\}}}(-1)^{u}\left|\operatorname{det}\left(I+F^{\left(n_{0}, n_{k_{1}}, \ldots, n_{k_{u}}\right)}\right)\right|\left|1-\omega^{\left(n_{0}, n_{k_{1}}, \cdots, n_{k_{u}}\right)}\right|\right) .
\end{aligned}
$$

Here the summation runs through the family of nonempty subsets $\left\{k_{1}, \ldots, k_{s}\right\}$ of $\{1, \ldots, t\}$. 
Theorem 7.5. Let $f$ be a self-map on $K$ of type $(F, \mathbf{b}, \omega)$. If $\omega \neq \pm 1$ is odd and $\operatorname{det}\left(I-F^{n}\right)=0$ but $\operatorname{det}\left(I+F^{n}\right) \neq 0$, then the prime Nielsen-Jiang periodic number of $f$ of period $n$ is

$$
\begin{aligned}
N \mathrm{P}_{n}(f)= & \frac{1}{2}\left(\left|\operatorname{det}\left(I+F^{n}\right)\right|\left|1-\omega^{n}\right|\right. \\
& +\sum_{\left\{k_{1}, \ldots, k_{s}\right\} \subset\{1, \ldots, t\}}(-1)^{s} \mid \operatorname{det}\left(I+F^{\left(n_{k_{1}}, \ldots, n_{k_{s}}\right)}|| 1-\omega^{\left(n_{k_{1}}, \ldots, n_{k_{s}}\right)} \mid\right),
\end{aligned}
$$

where the summation runs through the family of nonempty subsets $\left\{k_{1}, \ldots, k_{s}\right\}$ of $\{1, \ldots, t\}$.

\section{The Nielsen type numbers: weakly Jiang case}

In this subsection we will finish our evaluation of the Nielsen type number of weakly Jiang maps.

When $f^{n}$ is weakly Jiang with $N\left(f^{n}\right)=R\left(f^{n}\right)$, due to Theorems 3.2 and 3.3 and Corollary 5.2 we can immediately state what the Nielsen type numbers are. Namely,

Corollary 8.1 (Case $N\left(f^{n}\right)=R\left(f^{n}\right)$ ). Let $f$ be a self-map on $K$ of type $(F, \mathbf{b}, \omega)$ with $N\left(f^{n}\right)=R\left(f^{n}\right)$. Then

$$
N \Phi_{n}(f)=N\left(f^{n}\right), \quad N \mathrm{P}_{n}(f)=\sum_{m \mid n} \mu(m) N\left(f^{\frac{n}{m}}\right)
$$

where $N\left(f^{k}\right)=\left|1-\omega^{k}\right|\left(\left|\operatorname{det}\left(I-F^{k}\right)\right|+\left|\operatorname{det}\left(I+F^{k}\right)\right|\right) / 2$.

Now we will work for the case where $N\left(f^{n}\right)=0$. If this is the case, there are no essential classes and thus $N \mathrm{P}_{n}(f)=0$. We are left to find $N \Phi_{n}(f)$. For this we will use Theorem 3.3: $N \Phi_{n}(f)=\sum_{k \mid n} N \mathrm{P}_{k}(f)$.

Corollary 8.2 (Case $\omega=1$ ). Let $f$ be a self-map on $K$ of type $(F, \mathbf{b}, 1)$. Then, for all $n, N \Phi_{n}(f)=N \mathrm{P}_{n}(f)=0$.

Proof. Since $\omega=1, N\left(f^{k}\right)=0$ and thus $N \mathrm{P}_{k}(f)=0$ for all $k$; hence $N \Phi_{n}(f)=0$.

Corollary 8.3 (Case $\left.\operatorname{det}\left(I \pm F^{n}\right)=0\right)$. Let $f$ be a self-map on $K$ of type $(F, \mathbf{b}, \omega)$ such that $\operatorname{det}\left(I \pm F^{n}\right)=0$. Then $n$ is odd and $N \Phi_{n}(f)=N \mathrm{P}_{n}(f)=0$.

Proof. If $\operatorname{det}\left(I \pm F^{n}\right)=0$ then Corollary 5.3 states that $n$ is odd and $N\left(f^{k}\right)=0=N \mathrm{P}_{k}(f)$ for all odd $k$. By Theorem 3.3, we have

$$
N \Phi_{n}(f)=\sum_{k \mid n} N \mathrm{P}_{k}(f)=0
$$

The last identity follows from the fact that $n$ is odd and so its factors $k$ must be odd and $N \mathrm{P}_{k}(f)=0$. 
Corollary 8.4 (Case $\omega=-1$ and $n$ is even). Let $f$ be a self-map on $K$ of type $(F, \mathbf{b},-1)$. Then for all even $n$,

$$
N \mathrm{P}_{n}(f)=0, \quad N \Phi_{n}(f)=\sum_{\substack{m \text { odd } \\ m \mid n}} N \mathrm{P}_{m}(f)
$$

where

$$
N P_{m}(f)=\left\{\begin{aligned}
& \sum_{k \mid m} \mu(k) N\left(f^{m / k}\right) \text { when } \operatorname{det}\left(I-F^{n}\right) \neq 0, \\
& \text { or } \operatorname{det}\left(I-F^{n}\right)=0 \text { but } \operatorname{det}\left(I \pm F^{m}\right) \neq 0 ; \\
&\left|\operatorname{det}\left(I+F^{m}\right)\right|+\sum_{\left\{k_{1}, \ldots, k_{s}\right\} \subset\{1, \ldots, t\}}(-1)^{s} \mid \operatorname{det}\left(I+F^{\left(m_{k_{1}}, \ldots, m_{k_{s}}\right)} \mid\right. \\
& \text { when } \operatorname{det}\left(I-F^{m}\right)
\end{aligned}\right.
$$

Here the summation runs through the family of nonempty subsets $\left\{k_{1}, \ldots, k_{s}\right\}$ of $\{1, \ldots, t\}$.

Proof. If $\omega=-1$ then for all even $m, N\left(f^{m}\right)=0$ and thus as before $N \mathrm{P}_{m}(f)=0$. By Theorem 3.3,

$$
N \Phi_{n}(f)=\sum_{m \mid n} N P_{m}(f)=\sum_{\substack{m \text { odd } \\ m \mid n}} N P_{m}(f) .
$$

Since $n$ is even, by Corollary 5.3 both $\operatorname{det}\left(I-F^{n}\right)$ and $\operatorname{det}\left(I+F^{n}\right)$ cannot be zero. Let $m$ be odd and $m \mid n$; since $n$ is even, $m<n$ and $n / m$ is even. Since $n / m$ is even, $I \pm F^{m}$ is a factor of $I-F^{n}$.

Assume $\operatorname{det}\left(I-F^{n}\right) \neq 0$. Then $\operatorname{det}\left(I \pm F^{m}\right) \neq 0$. This is the case where $f^{k}$ is weakly Jiang with $N\left(f^{k}\right)=R\left(f^{k}\right)$ for any $k \mid m$. By Corollary 8.1,

$$
N \Phi_{n}(f)=\sum_{\substack{m \text { odd } \\ m \mid n}} N P_{m}(f)=\sum_{\substack{m \text { odd } \\ m \mid n}} \sum_{k \mid m} \mu(k) N\left(f^{m / k}\right) .
$$

Assume $\operatorname{det}\left(I-F^{n}\right)=0$.

CASE 1. $\operatorname{det}\left(I \pm F^{m}\right)=0$.

This happens only when the eigenvalues $\lambda_{i}$ of $F$ satisfy $\lambda_{1}=-\lambda_{2}= \pm 1$. Furthermore, $N\left(f^{m}\right)=0$ and so $N \mathrm{P}_{m}(f)=0$.

CASE 2. $\operatorname{det}\left(I \pm F^{m}\right) \neq 0$.

Then $f^{m}$ is weakly Jiang with

$$
N\left(f^{m}\right)=R\left(f^{m}\right)=2\left(\left|\operatorname{det}\left(I-F^{m}\right)\right|+\left|\operatorname{det}\left(I+F^{m}\right)\right|\right) .
$$


By Corollary 8.1,

$$
N \mathrm{P}_{m}(f)=\sum_{k \mid m} \mu(k) N\left(f^{m / k}\right) .
$$

CASE $3 . \operatorname{det}\left(I-F^{m}\right)=0 \neq \operatorname{det}\left(I+F^{m}\right)$.

By Theorem 6.6,

$$
N \mathrm{P}_{m}(f)=\left|\operatorname{det}\left(I+F^{m}\right)\right|+\sum_{\left\{k_{1}, \ldots, k_{s}\right\} \subset\{1, \ldots, t\}}(-1)^{s} \mid \operatorname{det}\left(I+F^{\left(m_{k_{1}}, \ldots, m_{k_{s}}\right)} \mid\right. \text {. }
$$

CASE 4. $\operatorname{det}\left(I-F^{m}\right) \neq 0=\operatorname{det}\left(I+F^{m}\right)$.

By Theorem 6.5,

$$
N \mathrm{P}_{m}(f)=\left|\operatorname{det}\left(I-F^{m}\right)\right|+\sum_{\left\{k_{1}, \ldots, k_{s}\right\} \subset\{1, \ldots, t\}}(-1)^{s} \mid \operatorname{det}\left(I-F^{\left(m_{k_{1}}, \ldots, m_{k_{s}}\right)} \mid\right.
$$

Consequently, all the observations give rise to our result.

\section{Summary}

We can tabulate where to find the formula for the prime Nielsen-Jiang pe-

\begin{tabular}{|c|c|c|c|c|}
\hline \multicolumn{2}{|c|}{$w$} & $n$ & $\operatorname{det}\left(I \pm F^{n}\right)$ & $N \mathrm{P}_{n}(f)$ \\
\hline even & & & & Corollary 8.1 \\
\hline \multirow{10}{*}{ odd } & 1 & & & Corollary 8.2 \\
\hline & \multirow{5}{*}{-1} & even & & Corollary 8.4 \\
\hline & & \multirow{4}{*}{ odd } & $\operatorname{det}\left(I \pm F^{n}\right)=0$ & Corollary 8.3 \\
\hline & & & $\begin{array}{l}\operatorname{det}\left(I-F^{n}\right) \neq 0 \\
\operatorname{det}\left(I+F^{n}\right)=0\end{array}$ & Theorem 6.5 \\
\hline & & & $\begin{array}{l}\operatorname{det}\left(I-F^{n}\right)=0 \\
\operatorname{det}\left(I+F^{n}\right) \neq 0\end{array}$ & Theorem 6.6 \\
\hline & & & $\operatorname{det}\left(I \pm F^{n}\right) \neq 0$ & Corollary 8.1 \\
\hline & \multirow{4}{*}{$\neq \pm 1$} & $\begin{array}{c}\text { odd } \\
\text { (Corollary } 5.3)\end{array}$ & $\operatorname{det}\left(I \pm F^{n}\right)=0$ & Corollary 8.3 \\
\hline & & & $\begin{array}{l}\operatorname{det}\left(I-F^{n}\right)=0 \\
\operatorname{det}\left(I+F^{n}\right) \neq 0\end{array}$ & Theorem 7.5 \\
\hline & & & $\begin{array}{l}\operatorname{det}\left(I-F^{n}\right) \neq 0 \\
\operatorname{det}\left(I+F^{n}\right)=0\end{array}$ & Theorem 7.4 \\
\hline & & & $\operatorname{det}\left(I \pm F^{n}\right) \neq 0$ & Corollary 8.1 \\
\hline
\end{tabular}
riodic number of $f, N \mathrm{P}_{n}(f)$, of maps $f$ on the flat Riemannian manifold $K$ as follows:

Acknowledgments. The authors would like to thank Xuezhi Zhao for helpful conversations on this project. We also would like to thank the referee for making careful corrections to a few expressions and suggesting some alternative approach in the original version of the article. 


\section{REFERENCES}

[1] H. Brown, R. Bülow, J. Neubüser, H. Wondratschek and H. Zassenhaus, Crystallographic Groups of Four-Dimensional Spaces, John Wiley \& Sons, Inc., New York, 1978.

[2] V.V. Gorbatsevich, Compact solvmanifolds of dimension at most 4, Siberian Math. J. 50 (2009), 239-252.

[3] K.Y. Ha, J.B. Lee And P. Penninckx, Formulas for the Reidemeister, Lefschetz and Nielsen coincidence number of maps between infra-nilmanifolds, Fixed Point Theory Appl., 2012, 2012:39.

[4] P.R. Heath, A survey of Nielsen periodic point theory, Nielsen theory and Reidemeister torsion (Warsaw, 1996), 159-188, Banach Center Publ., 49, Polish Acad. Sci., Warsaw, 1999.

[5] _ Fibre techniques in Nielsen theory calculations, Handbook of topological fixed point theory, Springer, Dordrecht, 2005, 489-554.

[6] P.R. Heath and E. Keppelmann, Fibre techniques in Nielsen periodic point theory on nil and solvmanifolds, R. Mat. Rep. Acad. Sci. Canada, 16 (1994), 229-234.

[7] _ Fibre techniques in Nielsen periodic point theory on nil and solvmanifolds I, Topology Appl., 76 (1997), 217-247.

[8] _ Fibre techniques in Nielsen periodic point theory on nil and solvmanifolds II, Topology Appl., 106 (2000), 149-167.

[9] B. JIANG, Lectures on Nielsen fixed point theory, Contemporary Mathematics Amer. Math. Soc., 14, 1983.

[10] E.C. Keppelmann And C.K. MCCond, The Anosov theorem for exponential solvmanifolds, Pacific J. Math., 170 (1995), 143-159.

[11] S.W. KIM, J.B. LEe AND K.B. LEe, Averaging formula for Nielsen numbers, Nagoya Math. J., 178 (2005), 37-53.

[12] H.J. Kim, J.B. Lee And W.S. Yoo, Computation of the Nielsen type numbers for maps on the Klein bottle, J. Korean Math. Soc. 45 (2008), 1483-1503.

[13] J.B. LeE AND K.B. LeE, Lefschetz numbers for continuous maps, and periods for expanding maps on infra-nilmanifolds, J. Geom. Phys. 56 (2006), 2011-2023.

[14] K.B. LeE, Maps on infra-nilmanifolds, Pacific J. Math. 168 (1995), 157-166.

[15] C. MCCord, Nielsen numbers and Lefschetz numbers on solvmanifolds, Pacific J. Math. 147 (1991), 153-164.

[16] J. Wolf, Spaces of Constant Curvature, 5th ed., Publish or Perish, Wilmington, 1984.

Ku Yong Ha And Jong Bum Lee

Department of Mathematics

Sogang University

Seoul 121-742, KOREA

E-mail address: kyha@sogang.ac.kr, jlee@sogang.ac.kr 